\title{
Melatonin Modulates Microglia Activation in Neuro- inflammation by Regulating the ER Stress/PPARd/SIRT1 Signaling Pathway
}

\section{Hung-Chuan Pan}

Taichung Veterans General Hospital

Cheng-Ning Yang

National Taiwan University

Wen-Jane Lee

Taichung Veterans General Hospital

Jason Sheehan

University of Virginia

Sheng-Mao Wu

National Chung Hsing University

Hong-Shiu Chen

Tungs' Taichung MetroHarbor Hospital

Mao-Hsun Lin

Ditmanson Medical Foundation Chia-Yi Christian Hospital

Li-Wei Shen

National Chung Hsing University

Shu-Hua Lee

National Chung Hsing University

Chin-Chang Shen

Atomic Energy Council

Liang-Yi Pan

Kaohsiung Medical University

Meei-Ling Sheu ( $\square$ mlsheu@nchu.edu.tw)

National Chung Hsing University

\section{Research Article}

Keywords: Melatonin, Microglia, Neuro-inflammation, PPARס, SIRT1

Posted Date: December 28th, 2021

DOl: https://doi.org/10.21203/rs.3.rs-1152032/v1 
License: (c) (i) This work is licensed under a Creative Commons Attribution 4.0 International License. Read Full License 


\section{Abstract}

Background: Activated microglia-mediated neuro-inflammation plays a vital aspect in regulating the micromilieu of the central nervous system. Neuro-inflammation involves distinct alterations of microglial phenotypes, containing nocuous pro-inflammatory (M1) phenotype and neuroprotective antiinflammatory (M2) phenotype. Currently, there is no effective treatment for modulating such alterations. Little evidence shows that melatonin prevents the detrimental cascade of activated microglia-mediated neuro-inflammation.

Methods: The expression levels of M1/M2 marker of primary microglia influenced by Melatonin were detected via qPCR. Functional activities were explored by western blotting, luciferase activity, EMSA, and ChIP assay. Structure interaction was assessed by molecular docking and LIGPLOT analysis. ER stress detection was examined by ultrastructure TEM, calapin activity, and ERSE assay. The neurobehavioral evaluations and immunofluorescence staining in animals were used for investigation of Melatonin on the neuroinflammation in vivo.

Results: Melatonin had targeted on Peroxisome Proliferator Activated Receptor Delta (PPARd) activity, boosted LPS-stimulated alterations in polarization from the M1 to the M2 phenotype, and thereby inhibited NFkB-IKKb activation in primary microglia. The PPARd agonist L-165041 or over-expression of PPARd plasmid (ov-PPARd) showed similar results. Molecular docking screening, dynamic simulation approaches, and biological studies of melatonin showed that the activated site was located at PPARd (phospho-Thr256-PPARd). Furthermore, we found that activated microglia had lowered PPARd activity as well as the downstream SIRT1 formation via enhancing ER stress. Melatonin, PPARd agonist and ovPPARd all effectively reversed the above-mentioned effects. Melatonin blocked ER stress by regulating calapin activity and expression in LPS-activated microglia. Additionally, melatonin or L-165041 ameliorated the neurobehavioral deficits in LPS-aggravated neuroinflammatory mice through blocking microglia activities, and also promoted phenotype changes to M2-predominant microglia.

Conclusions Melatonin suppressed neuro-inflammation in vitro and in vivo by tuning microglial activation through the ER stress-dependent PPARd/SIRT1 signaling cascade. We proposed that this treatment strategy is an encouraging pharmacological approach for the remedy of neuro-inflammation associated disorders.

\section{Introduction}

Microglia are known as resident macrophages in the central nervous system (CNS) and maintain its homeostasis by modulating phenotypic expressions. Microglial cells monitor the CNS microenvironment and respond to danger signals in physiological conditions.[1] Activated microglial cells undergo morphological transformation to ameboid, rod-shaped and ramified forms. They have expanded cell size, thickened proximal processes, and reduced ramification of distal branches. Activated microglia, when under influence of released inflammatory mediators, result in self-perpetuating damage to neurons. 
Microglia with such action are classically regarded as the activated pro-inflammatory M1 phenotype.[2, 3] Nonetheless, an another activated anti-inflammatory M2 phenotype can trigger the neuroprotective or neurosupportive effect, promoting recovery, and improving brain reparation or regeneration.[4] The status of microglia, may therefore obtains a battery of different, but super-imposed functional phenotypes.[5] In defining the microglia phenotype, the dichotomy of $M 1$ and $M 2$ is a useful notion for studying the detrimental or beneficial effect of the activated microglia, and it is likely helpful in exploring innovative strategies.

Peroxisome proliferator-activated receptor delta $(P P A R \beta / d)$ is nuclear receptor family of ligand-activated transcription factors. They heterodimerize with the retinoic $X$ receptor (RXR) to handle gene expressions likely in fatty acid oxidation, cholesterol metabolism, and thermogenesis.[6] They further form heterodimers with the RXR and also bind to PPAR response elements (PPREs) targeted on the promoter genes. Together they harness a variety of biological processes for the occurrence of chronic diseases, including caner, atherosclerosis, diabetes, and obesity.[7] Growing evidence indicates that PPARd agonists are exercise mimetic, and they reduce glucose utilization in isolated skeletal muscles through switching mitochondrial substrate preference from carbohydrate to lipid.[8, 9] In addition, PPAR $\beta / \delta$ ligand suppresses inflammation and promotes neovascularization during corneal wound healing process.[10] Activation of peroxisome proliferator-activated receptor delta by GW501516 generates fatty acid betaoxidation in skeletal muscles and lessening metabolic syndrome, thereby attenuating obesity and insulin resistance in obese animals.[11] Also, activation of PPARd serves as a therapeutic target for ameliorating inflammation and slowing the progression of atherosclerosis.[12] In HFD-fed mice, chronic peroxisome proliferator-activated receptor beta/delta agonist GW0742 prohibited early manifestations of vascular inflammatory and oxidative status, atherosclerosis, endothelial dysfunction and, hypertension.[13] Thus, $\mathrm{PPAR} / \delta$ triggering not only prevents obesity, augment protective effects on hypertension and atherosclerosis, but also reduces vascular pro-oxidant and pro-inflammatory status.

Endoplasmic reticulum (ER) stress is involved in neuronal changes caused by infection/inflammation, hypoxia, ischemia or reperfusion (IR) injury. Suppression of ER stress protects against neuronal injuries. [14-17] Several studies reported that ER stress-associated inflammation modifies microglial or macrophage activation in traumatic brain injury or ischemic stroke. $[17,18]$ In addition, ER stress changes the autophagic response or modulates the ER stress-autophagy axis which induces cytotoxicity in microglial cells. $[17,19]$ Several studies also reported that melatonin positively regulates both ER stress and autophagy, and alleviates ER stress induced neonatal brain inflammation or neurotoxicity.[20] The latest investigations have confirmed that ER stress contributes to the microglial activation process, which causes neuro-inflammation.[21-23] Moreover, the action of Sirtuin 1, (SIRT1) is neuroprotective and its role, as shown in the retrograde degenerative progress in spinal cord motoneurons (MNs) subjected to root avulsion (RA) injury, is highly correlated with ER stress.[24] However, the mechanism of SIRT1in relieving ER-induced microglia activation is not fully understood.

Melatonin ( $\mathrm{N}$-acetyl-5-methoxytryptamine) is predominately released by the enigmatic pineal gland, and serotonin is regarded as its precursor, a neurotransmitter derived from the amino acid L-tryptophan. 
[25] Serotonin is acetylated and then methylated to form melatonin within the pineal gland.[26] Melatonin operates through $\mathrm{G}$ protein-coupled receptors $\mathrm{M} 1$ and $\mathrm{M} 2$ as well as modulates the circadian rhythms and enhances the immunity. It not only acts as a multifunctional oncostatic agent and antioxidant, but also exerts anti-inflammatory, and antidepressant effects. The antecedent studies reported that melatonin markedly inhibits the LPS-induced pro-inflammatory cytokines such as TNFa, IL-6, and IL1b.[2730] Moreover, melatonin reduces oxidative stress, mitochondrial toxicity, induces autophagy and inhibits apoptosis.[27, 29-35] Our previous study in cancer therapy demonstrated melatonin effectively stimulates the ER stress pathway to thwart epithelial mesenchymal transition and peritoneal dissemination. [36] However, the molecular mechanisms of melatonin in neuro-inflammation-induced microglia inactivation and relief of ER stress are yet to be well studied.

Here, we aimed to evaluate the deleterious response of microglial activation by LPS administration. Such a response was rescued by neuroprotective effects of melatonin. We intended to investigate how melatonin attenuates LPS-induced neuro-inflammation and alleviates the impairments due to ER stress. Our results showed that melatonin treatment markedly abolished the LPS-evoked switch of microglial polarization from the M1 to M2 phenotype. Melatonin also reduced the ER stress and its associated proinflammatory mediators via the PPARd/SIRT1 related signaling pathway. Melatonin may therefore be a potent therapeutic strategy to treat neuro-inflammation-related diseases.

\section{Materials And Methods}

The methods listed below have been published before but are repeated here for clarity and shown in Supplementary material methods.[22, 23, 36]

\section{Chemicals and materials}

LPS (Escherichia coli 0111: B4) was purchased from Sigma-Aldrich. Melatonin was also purchased from Sigma-Aldrich. L-165041 is a phenyloxyacetate PPARס receptor agonist from R\&D. The calpain inhibitors were obtained from BIOMOL and from Merck Biosciences.

\section{Cell culture}

Microglia were extracted from the Sprague-Dawley rats aged 1-3 days and cultured according to procedures previously described.[22, 23, 36] In brief, culture media were first replaced 2 days after preparation, and media were replaced every 2 to 3 days thereafter. Before the experiment, the percentages of the primary rat microglial cells were estimated with Iba1 staining, and confirmed $>97 \%$ in purity.

\section{RNA isolation and quantitative PCR}


To study gene expression, total RNA was extracted from BV2 cell lines, primary microglia culture, or brain cortical tissue using the Trizol reagent. The isolated and purified RNA was reverse-transcribed into cDNA using cDNA synthesis kit according to standard protocols. Quantitative PCR (qPCR) was done using synthetic primers and SYBR Green on a Bio-Rad iQ5 Multicolor Real-Time PCR Detection System. Incubated at $50{ }^{\circ} \mathrm{C}$ for $2 \mathrm{~min}$ and $95^{\circ} \mathrm{C}$ for $10 \mathrm{~min}$, target samples were allowed to 35 cycles at $95^{\circ} \mathrm{C}$ for 15 secs and $60^{\circ} \mathrm{C}$ for $1 \mathrm{~min}$. Table I showed the list of qPCR primers. The data were presented as the fold-change of the target gene relative to GAPDH.

\section{Immuno-blotting}

Whole-cell lysates of microglia were prepared as previously described for immuno-blotting.[22, 23, 36] Antibodies used are shown in Supplementary Tables 1 and 2. Assessment was conducted using ECL (Amersham) and by chemiluminescence on Kodak X-Omat film. The data was repeated 5 times independently.

\section{Transmission electron microscopy (TEM)}

The procedure was executed previously described.[22, 23, 36] The targeted samples under different treatment were fixed with $4 \%$ glutaraldehyde and then $2.5 \%$ paraformaldehyde dissolved in $0.1 \mathrm{M}$ sodium cacodylate, and then further post-fixed in 1\% osmium tetraoxide, dehydrated in ethanol, and embedded in araldite. Sections on grids were counter-stained with uranyl acetate and lead citrate, and images validated with a JEM 1200 EX TEM (JEOL, Peabody, MA, USA) at an accelerating voltage of $80 \mathrm{kV}$.

\section{Electrophoretic mobility shift assay (EMSA), Chromatin immunoprecipitation (ChIP) assay}

The oligonucleotide with the PPARd consensus binding sequence used was PPARd/SIRT1 -384 -345 (50CGGCCAGGTTGGGCTCTCCAGAGCCCCTGAGGTAAAAGCC-30). DNA-protein complexes were resolved on $6 \%$ nondenaturing polyacrylamide gels, and visualized by exposing the autoradiographic films. The modified ChIP assay protocol was depicted previously. We used a fragment (200 b.p.) of the SIRT1 promoter containing putative PPARd -binding sites. The forward primer was _642 to _661 (50-5'TCGGCTGATCTCCAAACCTC-3'-3), while the reverse primer was 50 to 70 (5'-CTCGCCACAAAGAGGAAGGG$\left.3^{\prime}\right)$. Thirty cycles of PCR were conducted at $94{ }^{\circ} \mathrm{C}$ for 30 seconds, at $64.8^{\circ} \mathrm{C}$ for 30 seconds, and at $72{ }^{\circ} \mathrm{C}$ for 45 seconds. PCR reactions were precipitated and run on a $1.5 \%$ agarose gel.

\section{Statistical analyses}


The calculated data were presented as mean \pm standard deviation. All the data were analyzed by analysis of variance (ANOVA) and post hocked by Fisher's least significant difference test. Statistical significance was set at $p<0.05$.

\section{Results}

\section{Melatonin reciprocally suppresses proinflammatory mediators and augments anti-inflammatory response in LPS-treated mouse primary microglia}

First, we handle LPS-induced primary microglia cells to check the power of melantonin in the antiinflammatory action. Melatonin showed no signs of toxicity on microglia at concentrations up to $1 \mathrm{mM}$ (data not shown). Treatment with melatonin significantly inhibited LPS-induced microglial activation (Figure.1A). Moreover, it dramatically diminished mRNA levels of M1 markers, such as CD86, COX-2, iNOS, IL-1b, IL-6, TNF-a, and CCL2, compared with the LPS groups (Figure.1Aa-h). IFNg showed opposite effects. Furthermore, expressions of M2 markers, like CD206, IL-4, GM-CSF, Arginase-1, IGF-1, TGF-b1, YM1/2, and IL-10 increased after melatonin application (Figure1Ai-p). Results showed that melatonin enhanced microglial M2 polarization. NF-KB is a main transcription factor of M1 macrophages, and it is essential for the ignition of a series of inflammatory associated genes or mediators. It is also crucial I for both M1- and M2-like microglial differentiations. After LPS-treated, we found remarkable increases in the phosphorylation of IKKb and NFKB, but downregulation of IKB. In contrast, melatonin forcefully abolished LPS-induced phosphorylations of IKKb and NFKB (Figure.1Ba). Quantitative results are shown in Figure $1 \mathrm{Bb}-\mathrm{d}$. Moreover, the luciferase-based approach for in vivo measurement of NF-KB promoter activity also showed that melatonin markedly blocked NF-KB activity (Figure.1Be). These findings suggested that the IKKb and NFKB participated in the phenotypic shift of microglial polarization through inhibition of IKKb/NFKB pathway.

\section{Agonizing PPARd using L16504, or overexpression of PPARd promotes M1-to-M2 polarization of primary cultured microglia}

A number of reports have displayed the important roles of PPARa and PPARg in microglial-induced inflammation and phenotype shift. Yet, how PPARd alters LPS-induced microglial polarization in the activated microglial remains unclear. In addition, simultaneous high-throughput screening with mass spectrometry showed that PPARd had played a crucial role for microglia activation. Here, to elucidate how to regulate activated microglia, we used the pharmacological induction method based on PPARd specific agonist L165041 and gene overexpression PPARd plasmid (ovPPARd). L165041 and ovPPARd were found to inhibit LPS-induced M1 microglial polarization in mouse primary microglia. The LPS treatment accelerated mRNA expression of inflammatory cytokines (M1 markers, CD86, iNOS, COX-2, IL-1b, IL-6, 
TNF-a, and CCL2), and pretreatment with melatonin attenuated mRNA expression of these inflammatory cytokines (Figure.2Ba-h). IFNg on the other hand, showed the opposite effects. In mouse primary microglia, LPS-stimulated microglial polarization was associated with a drop in mRNA expressions of M2 markers (CD206, IL-4, GM-CSF, Arginase-1, IGF-1, TGF-b1, YM1/2, IL-10). However, melatonin pretreatment elevated mRNA expressions of all the aforementioned markers (Figure.2Bi-p). In addition, ELISA showed that melatonin treatment enhanced the release of IL-10 in LPS-treated mouse primary microglia (data not shown). Melatonin suppressed M1 microglia polarization, promoted M2 microglia polarization, and attenuated inflammatory responses in activated microglia. According to the above-mentioned findings, $\mathrm{NF}-\mathrm{KB}$ pathways are involved in M1 polarization roles and involved in the regulation of microglial activation. After LPS induction, marked enhancement in phosphorylation was found in IKKb and NFKB, but marked decrement was found in IKB. In contrast, L165041 or transfection of ovPPARd plasmid significantly inhibited LPS-induced phosphorylation of IKKb and NFKB (Figure.2Ba-b). The quantitative results are shown in Figure.2Bc-e. Moreover, NF-KB luciferase activity was markedly blocked by L165041 and the transfection of ovPPARd. These findings suggested that PPARd activation participated in the phenotypic shift of microglial polarization by suppressing IKKb/NFKB pathway.

\section{Molecular docking, dynamic simulation studies and biochemical evidence show activation properties of Melatonin targeting PPARd.}

Molecular docking emerges as a paramount tool for drug discovery, and it can miniature interactions between small molecules and ligand-proteins at the atomic level. That yields the characterization of their interacting behavior as well as their biochemical processes of known three-dimensional structure. Dynamic simulation based on a computer program allows the evaluation and understanding of transient physical and chemical processes of the time-varying behavior. In the present study, we used the approaches of both docking and dynamic simulation to investigate the potential interactions between melatonin and PPARd. Results appeared that the key residues within the PPARd catalytic site had interacted with melatonin, and the binding took place in the tunnel of the active site (Figure.3A-B). Crystal structures of PPARd showed that the highly conserved Thr256 residue in PPARd was located within the active site, which was catalytically required in stabilizing the functional activity of the molecule. Changes in both conformation and dynamics likely occurred when melatonin was bound to PPARd, facilitating melatonin for targeting the active site. A computer program, bioinformatics LIGPLOT, generates schematic 2-D representations of protein-ligand complexes. It showed that interplay with the candidate site contained the Thr256 residue (Figure.3C). Furthermore, specific antibody targeting phosphorylation of PPARd (Thr 256) in microglia was markedly induced by melatonin after 30 minutes of treatment (Figure.3D). The response gradually lessened after 60 minutes. Importantly, L165041 or transfection of ovPPARd evoked phospho-Thr256-PPARd as detected by western blotting. Moreover, LPS-induced phospho-PPARd (Thr256) translocated to the nucleus was enhanced markedly by melatonin induction as shown in confocal fluorescence images (Figure.3E). In summary, these results implicated a possible 
interaction between melatonin and PPARd based on structure molecular docking as well as phosphoPPARd (Thr256) activities. Melatonin is therefore a direct target of phospho-PPARd (Thr256), and our methods worked well in predicting interactions of melatonin with its target ligand protein PPARd.

\section{Melatonin-evoked PPARd activity regulated SIRT1 production.}

PPARd agonists have potential neuroprotective effects. Beneficial roles of SIRT1 are known in neuroinflammation-related diseases. We aimed to predict in silico promoter binding sites to elucidate possible mechanisms. The SIRT1 promoter-flanking region (-345 -384) containing the cis-acting elements PPARd DNA binding activity in silico was predicted in the laboratory (Figure.4A). Simultaneously, we detected PPARd and SIRT1 protein co-expressions. LPS-induced PPARd and SIRT down-regulations were detected in western blotting (Figure.4B). To determine whether Melatonin evoked transcriptional activity of microglia, the expression of transcriptional activity was assessed by luciferase reporter assay (Figure.4C). LPS reduced PPARd activity, which could be reversed by melatonin. In addition, our previous study showed that apoptosis episode in LPS-induced microglia is regulated by calpain-II activity under conditions of ER stress.[22] In that study, exposures to Calpain inhibitor (Z-Val-Phe-CHO) also markedly reversed PPARd expressions. These suggested a strong standing point of PPARb in transcriptional activity in response to LPS-induced ER stress in activated microglia. For the purpose to demonstrate that PPARb directly regulates SIRT1 expression, DNA binding activity of the SIRT1 promoter was measured. As shown in Figure.4D, EMSA assay showed that LPS had suppressed PPARd binding activity at the SIRT1 promoter binding site across time frames. Noticeably, pretreatment with melatonin enhanced DNA binding activity (Figure.4D; left). On the other hand, pretreatment with PPARd agonist L165041 or transfection with PPARd (ovPPARd) intensified similar PPARd binding activity (Figure.4D; right). In ChIP experiments with an anti-PPARd polyclonal antibody, PCR amplification at the specific PPARd locus of the SIRT1 promoter obtained with chromatin purified from control cells gave a strong signal. In contrast, controls with immunoprecipitation in normal rabbit serum (IgG) gave only a weak background signal. In LPS-treated microglia, the signal reduced gradually after $1 \mathrm{hr}$. But melatonin pre-treatment had selectively enriched specific DNA-binding proteins PPARd along with their DNA targets SIRT1 (Figure.4E). We further studied the aspect of PPARd in the amplitude of SIRT1 and the regulatory relationship between PPARd and the calpain signals. As shown in Figure.4F, microglia treated with LPS resulted in lowered expression of SIRT1 protein. Melatonin or PPARd agonist L165041 effectively increased the expression of SIRT1. Moreover, the calpain inhibitor Z-Leu-Leu-CHO markedly enhanced the expression. The transfection of ovPPARd in activated microglia also led to a significant induction of the SIRT1 protein expression. These results implied that melatonin-enhanced PPARd regulated SIRT1 signaling pathway involved in LPStriggered microglial activation. Our findings reinforced the hypothesis that melatonin tuned the PPARd binding to the SIRT1 promoter via the calpain signaling cascade. 


\section{Melatonin circumvented ER stress-related markers in LPS- activated microglia}

To directly investigate the effects of melatonin on activated microglia by ER stress induction, we assessed the morphology of ER ultrastructures using the transmission electron microscopy (TEM). As shown in Figure.5A, we found dilated ER in LPS-treated cells. Pretreatment of melatonin rescued the effect, seen as less distention and fragmented organelles. Moreover, on exposure to L165041, we found similar phenomenon. We next examined the response of ER stress-related unfolded proteins in activated microglia with or without melatonin or L165041. As shown in Figure.5B, LPS exaggerated the response in phospho-PERK, phospho-elF2a and GADD153 in primary and BV-2 microglial cells. Melatonin or L165041 markedly lowered expressions of above said ER stress associated markers in activated microglia. Melatonin or L165041 fundamentally had no appreciable effect on ER stress associated markers (data not shown). We further demonstrated that PPARd antagonist GSK3787 combined with LPS had aggravated the activated microglia (Figure.5B). Additionally, we examined ER stress activity using the ER stress-response elements (ERSE) reporter system. We found that LPS induced ER stress activity, and melatonin completely abolished the reporter activity. On exposure to L165041 (10 mM), the downregulation effect was observed (Figure.5C). However, GSK3787 (5 mM) alone or in combination with LPS-induction enhanced ER stress activity. Tunicamycin treatment was taken as a positive control for the ER stress induction. These results indicated that melatonin had blocked ER stress in activated microglia.

\section{Melatonin blocked induction of calpain protein and activity in LPS-activated microglia.}

Calpain is renowned for the involvement of ER stress induced neurodegeneration. First, we examined whether melatonin blocked the calpain activity in activated microglia and its associated calpain protein expressions. As shown in Figure.6A, LPS increased calpain activity in primary microglia, starting at 30 minutes and beyond. Combined with calpain inhibitor (Z-Leu-Leu-CHO; $10 \mathrm{mM}$ ), LPS markedly reduced calpain activity. LPS also increased microglial calpain activity, and that was significantly reduced by melatonin or L165041. However, the antagonist GSK3787 showed no effect (Figure.6B). In addition, transfection of ovPPARd attenuated calpain activity. Gene silencing of PPARd by shPPARd on the other hand, produced no effect (Figure.6C). Regarding expressions of calpain associated proteins, Calpain-I and Calpain-II were induced in LPS-activated microglia, and their inductions were reversed by melatonin (Figure.6D). We further examined whether melatonin suppressed the interaction between Calpain and PPARd. As shown in Figure.6E, the interaction of Calpain with PPARd (as shown by immunoprecipitation assay), which was modest in the control cells after LPS stimulation, became significantly lower for 90 minutes in the presence of melatonin. The $10 \%$ input displayed for protein visualization was used as a positive control for the comparison. In addition, confocal microscopy revealed greater co-localization of Calpain-I/II with PPARd in microglia after LPS exposures for 2-4 hr (Figure.6F). Such co-localization was 


\section{Melatonin therapy restrains microglia activation and enhances PPARd expression.}

Inflammatory conditions and nerve injuries alter gait, suppress general locomotion and voluntary wheel running, reduce social interactions, and/or induce anxiety-like behavior.[37] To assess in-vivo effects of melatonin and L165041 in the activated microglia, we conducted neurobehavioral evaluations using Ethovision, Rotarod, and CatWalk gait analysis. In Ethovision assessments, either in the short term and long-term memory, we found that LPS injections aggravated the neurobehavior. Either melatonin or L165041 had alleviated such aggravations (Figure.7A;7C). Gross motor function tests (rotarod), related to motor coordination and balance, also showed similar trends (Figure.7B). In the Catwalk gait analysis, deterioration of gait balance was triggered by LPS injection, and improvement was found after melatonin and L165041 injections (Table4). To further investigate whether administration of melatonin exhibits neuroprotective effects in rats with activated microglia, the extent of inflammation was assessed with immunofluorescence staining. On day 7 after LPS-induction, the sham group showed only minimal sized inflammatory areas (Figure.7D). Compared with the LPS group, melatonin ( $5 \mathrm{mg} / \mathrm{kg}$, i.p.) significantly reduced the overwhelmingly activated microglia volume $(P<0.01)$ (Figure.7E). Simultaneously, we also found similar effects with the PPARd agonist L165041 (2 mg/kg, i.p.) (Figure.7F). Quantification is shown in Figure.7G. This finding suggested that melatonin uniquely reduced neuroinflammatory conditions in activated microglia, via a PPARd regulatory mechanism.

\section{Discussion}

Emerging evidence indicates that down-regulation of microglial proinflammatory mediator's expressions and augmentation of the anti-inflammatory factors production both play a pivotal role in neuroprotective recovery after brain injury. Our study was therefore designed to determine whether melatonin, secreted by the pineal gland in the brain, has anti-neuroinflammatory effects in vitro and in vivo based on a LPSinduced neuro-inflammation animal model. The current functionally polarized classification of microglia into $\mathrm{M} 1$ or $\mathrm{M} 2$ remains a subject of debate. The $\mathrm{M} 1 / \mathrm{M} 2$ polarization of microglia is largely based on genes associated with M1 markers (CD86, COX-2, iNOS, IFNg, IL-1b, IL-6, TNF-a, CCL2) and M2 markers (CD206, IL-4, GM-CSF, Arginase-1, IGF-1, TGF-b1, YM1/2, IL-10). In response to sensing distinct microenvironmental cues, the polarized M1 and M2 subpopulations can reverse their phenotype and biological functions. Forthcoming evidence revealed that ER stress is essential for the induction and functional alteration in M1 phenotype microglia. Therefore, strategies based on stimulating M1-to-M2 phenotype conversions are potentially useful in treating neuro-inflammation-induced injuries. The main findings of our study are as follows: 1) Melatonin inhibits microglial activation, promotes M2 polarization, and suppresses inflammatory mediators. 2) Melatonin reduces activated microglia, improves sensorimotor recovery and inhibits the proinflammatory mediators in a mouse model, and 
ameliorates deficiency of neurobehavior in a LPS-induced neuro-inflammation animal model. 3) Beneficial effects of melatonin-induced PPARd activation in regulating downstream SIRT1 in vivo are associated with the anti-ER stress and anti-inflammatory effects. 4) We further obtained evidence that the application of the PPARd agonist L165041 facilitates microglial phenotypic polarization from M1 to M2 and contributes to the anti-inflammatory effects (Fig. 8).

Microglia represent an important element of the immune system's inflammatory response in the CNS, and they inhibit or promote inflammation dependent on different conditions. Therefore, microglia plays dual roles either in detrimental or beneficial for functional recovery. In our previous study, we found that IL-13 downregulates PPAR-gamma/heme oxygenase-1 via ER stress-stimulated calpain in activated microglia responding to endotoxin injury.[22] Furthermore, the ER stress-related calpain, downregulates the PPARY/HO-1 pathway via C/EBP- $\beta$ and that leads to aggravated death of activated microglia via IL-13. Consequently, the results contributed to the prevention of cerebral inflammation and neuronal injury.[23] Regarding anti-inflammatory effects of melatonin on activated microglia, we studied mouse primary microglia cells or BV2 cells activated by LPS. We found that melatonin had enhanced expression of antiinflammatory mediators like PPARd and SIRT1. Melatonin is known to up-regulate SIRT1 during aging, and it displays pro-oxidant, pro-apoptotic and SIRT1-downregulating properties in cancer cells.[28, 38-40] Meanwhile, a connection between melatonin and another mediator component, SIRT1, is becoming clearer; SIRTuin may also mediate other effects.[28, 39, 40] Furthermore, in PND7 rat brains, melatonin treatment rescues the hippocampal dentate gyrus (DG) against LPS-induced oxidative stress damage, acute neuro-inflammation, and apoptotic neurodegeneration via the SIRT1/Nrf2 signaling cascade.[29] Furthermore, melatonin through the SIRT1 pathway attenuates LPS-induced acute depressive-like behaviors and microglial NLRP3 inflammatory activation.[41] Consistent with these effects, melatonin also acts as an antidepressant via attenuation of neuro-inflammation by targeting SIRT1 signaling.[42] In this study, we found that SITR1 expression was also markedly increased after melatonin-induction, a finding which is consistent with all previous findings. However, we provided new information on gene regulation for the transcription factor PPARd. Our results indicated that the neuroprotective effects of melatonin in brain damage is, at least in part, associated with the microglial anti-inflammatory activation via the PPARd/SIRT1 signaling pathway.

PPAR activation regulates the inflammatory activity mediated by microglia and astrocyte, protecting neurons from insult, reducing oxidative stress, inhibiting ER stress, and improving mitochondrial functions. In particular, reports from our previous study and from others showed that PPARg is mainly expressed in microglia, and it mediates anti-inflammatory activity by inhibiting oxidative stress, reducing ER stress, and inducing microglial polarization to M2-like phenotype. Melatonin attenuates dysregulation of the circadian clock pathway in mice with ccl4-induced fibrosis and human hepatic stellate cells through PPARa activation.[43] PPAR-a modulates the anti-inflammatory effect of melatonin in the secondary events of spinal cord injury under PPARa KO mice. This indicates that PPAR-a can contribute to the anti-inflammatory activity of melatonin in SCl.[44] On the other hand, melatonin significantly increases the expression of PPAR- $y$, a master regulator of adipogenesis, and it promotes cell differentiation into adipocytes.[45] Melatonin attenuates acute pancreatitis-induced liver damages 
through AKT-dependent PPAR-y Pathway.[46] Melatonin suppresses platelet activation and function against cardiac ischemia/reperfusion injury via PPARY/FUNDC1/mitophagy pathways. This action may provide a novel strategy for cardioprotection in cardiac l/R injury. ${ }^{50}$ Currently, there is very little known about the correlation between melatonin and PPARd. To characterize the role of PPARd in melatoninmediated anti-inflammatory activity, we tested the efficacy of melatonin $(10 \mathrm{mg} / \mathrm{kg})$ in an experimental model of LPS-induced neuro-inflammation using functional study and histology images to examine activation of microglia response. We found that melatonin markedly reduced the deterioration in neurobehavior and attenuated microglia cells activation. The PPARd agonist, L165041, also possessed similar effects. Based on the structural biology approach, we constituted the melatonin target PPARd (Thr256 site) through molecular docking. In the in vitro study, melatonin activated phospho-PPARd in the early stage, and forward PPARd to regulate SIRT1 protein expressions. Therefore, PPARd is likely a novel therapeutic target for neurodegenerative diseases. In the present study, we reported, for the first time, that melatonin induces PPARd activation, and that PPARd played a critical role as a master regulator of antiinflammatory action. Our findings supported the therapeutic potential of melatonin in treating neurodegenerative diseases or neuro-inflammation disorders.

\section{Conclusion}

Melatonin, PPARd and its agonist L165041 reduced neurobehavioral deficits induced by LPS injections, exerting anti-inflammatory effects and thwarting ER stress, through the PPARd/SIRT1 signaling pathway. Melatonin and PPARd and its agonist L165041 hopefully are encouraging pharmacological candidates for treating brain disorders associated with neuro-inflammation.

\section{Abbreviations}

CNS: Central Nervous System; Chip; Chromatin Immunoprecipitation (Chip) Assay: ER: Endoplasmic Reticulum; EMSA: Electrophoretic Mobility Shift Assay; HFD High-Fat Diet: IR: Ischemia Or Reperfusion; KO Mice: Knock-Out Mouse LPS: Lipopolysaccharides; TEM: Transmission Electron Microscopy; Ov-PPARd: Over-expression of PPARd Plasmid; PPARd: Peroxisome Proliferator Activated Receptor Delta; RXR: Retinoic X Receptor; RA: Root Avulsion; shRNA: Short Hairpin RNA; SIRT1: Sirtuin 1; PPRES: PPAR Response Elements; QPCR: Quantitative PCR; MNs: Motoneurons.

\section{Declarations}

\section{Consent for publication}

All authors have given their consent for publication.

\section{Availability of data and materials}


All data supporting the conclusions of this study are presented in the manuscript. The data sets analyzed for the current study are available from the corresponding author upon a reasonable request.

\section{Competing interests}

The authors declare that they have no competing interests.

\section{Funding}

This study was supported by grants from the Ministry of Science and Technology, the National Science Council, Taiwan (MOST-106-2320-B-005-001-MY3, MOST-104-2320-B-005-002-MY3), National Chung Hsing University, Taiwan (NCHU108ST001F), Taichung Veterans General Hospital in Taiwan (TCVGH1087327D, TCVGH-1087308C, TCVGH-1077311C, TCVGH-1077329D) and Tungs' Taichung MetroHarbor Hospital, Taichung, Taiwan

\section{Authors' contributions}

Pan HC, Wu SM and Sheu ML performed all of the mouse and cellular experiments, and generated and analyzed data; Yang CN, Pan LY for primary all of mouse primary microglia and BV2 microglia. Lee WJ, Sheehan $\mathrm{J}$ consulted and analyzed the data. Pan HC, Lin MH and Chen HS provide clinical information. Lee SH participated in immunofluorescence image experiments. Shen CC, Shen LW conducting molecular docking studies and LIGPLOT. Sheu ML directed the experiments and analyzed and assembled the data. All authors approved the final manuscript.

\section{Acknowledgments}

Precision instruments center, core facility and animal center of Taichung Veterans General Hospital in Taiwan. National RNAi Core Facility Platform, National Core Facility for Biopharmaceuticals, Academia Sinica, Taipei, Taiwan.

\section{References}

1. Saijo K, Glass CK. Microglial cell origin and phenotypes in health and disease. Nature reviews Immunology. 2011;11(11):775-87. Epub 2011/10/26. doi: 10.1038/nri3086. PubMed PMID: 22025055.

2. Bohatschek M, Kloss CU, Kalla R, Raivich $\mathrm{G}$. In vitro model of microglial deramification: ramified microglia transform into amoeboid phagocytes following addition of brain cell membranes to microglia-astrocyte cocultures. Journal of neuroscience research. 2001;64(5):508-22. Epub 2001/06/08. doi: 10.1002/jnr.1103. PubMed PMID: 11391706. 
3. Sochocka M, Diniz BS, Leszek J. Inflammatory Response in the CNS: Friend or Foe? Molecular neurobiology. 2017;54(10):8071-89. Epub 2016/11/28. doi: 10.1007/s12035-016-0297-1. PubMed PMID: 27889895; PubMed Central PMCID: PMCPMC5684251.

4. Atri C, Guerfali FZ, Laouini D. Role of Human Macrophage Polarization in Inflammation during Infectious Diseases. International journal of molecular sciences. 2018;19(6). Epub 2018/06/21. doi: 10.3390/ijms19061801. PubMed PMID: 29921749; PubMed Central PMCID: PMCPMC6032107.

5. Chhor V, Le Charpentier T, Lebon S, Oré MV, Celador IL, Josserand J, et al. Characterization of phenotype markers and neuronotoxic potential of polarised primary microglia in vitro. Brain, behavior, and immunity. 2013;32:70-85. Epub 2013/03/05. doi: 10.1016/j.bbi.2013.02.005. PubMed PMID: 23454862; PubMed Central PMCID: PMCPMC3694309.

6. Aleshin S, Strokin M, Sergeeva M, Reiser G. Peroxisome proliferator-activated receptor (PPAR) $\beta / \delta$, a possible nexus of PPARa- and PPARy-dependent molecular pathways in neurodegenerative diseases: Review and novel hypotheses. Neurochemistry international. 2013;63(4):322-30. Epub 2013/07/03. doi: 10.1016/j.neuint.2013.06.012. PubMed PMID: 23811400.

7. Juge-Aubry C, Pernin A, Favez T, Burger AG, Wahli W, Meier CA, et al. DNA binding properties of peroxisome proliferator-activated receptor subtypes on various natural peroxisome proliferator response elements. Importance of the 5 '-flanking region. The Journal of biological chemistry. 1997;272(40):25252-9. Epub 1997/10/06. doi: 10.1074/jbc.272.40.25252. PubMed PMID: 9312141.

8. Brunmair B, Staniek K, Dörig J, Szöcs Z, Stadlbauer K, Marian V, et al. Activation of PPAR-delta in isolated rat skeletal muscle switches fuel preference from glucose to fatty acids. Diabetologia. 2006;49(11):2713-22. Epub 2006/09/09. doi: 10.1007/s00125-006-0357-6. PubMed PMID: 16960684.

9. Narkar VA, Downes M, Yu RT, Embler E, Wang YX, Banayo E, et al. AMPK and PPARdelta agonists are exercise mimetics. Cell. 2008;134(3):405-15. Epub 2008/08/05. doi: 10.1016/j.cell.2008.06.051. PubMed PMID: 18674809; PubMed Central PMCID: PMCPMC2706130.

10. Tobita Y, Arima T, Nakano Y, Uchiyama M, Shimizu A, Takahashi H. Peroxisome Proliferator-Activated Receptor Beta/Delta Agonist Suppresses Inflammation and Promotes Neovascularization. International journal of molecular sciences. 2020;21(15). Epub 2020/07/30. doi: 10.3390/ijms21155296. PubMed PMID: 32722564; PubMed Central PMCID: PMCPMC7432070.

11. Tanaka T, Yamamoto J, Iwasaki S, Asaba H, Hamura H, Ikeda Y, et al. Activation of peroxisome proliferator-activated receptor delta induces fatty acid beta-oxidation in skeletal muscle and attenuates metabolic syndrome. Proceedings of the National Academy of Sciences of the United States of America. 2003;100(26):15924-9. Epub 2003/12/17. doi: 10.1073/pnas.0306981100. PubMed PMID: 14676330; PubMed Central PMCID: PMCPMC307669.

12. Lee $\mathrm{CH}$, Chawla A, Urbiztondo N, Liao D, Boisvert WA, Evans RM, et al. Transcriptional repression of atherogenic inflammation: modulation by PPARdelta. Science (New York, NY). 2003;302(5644):4537. Epub 2003/09/13. doi: 10.1126/science.1087344. PubMed PMID: 12970571. 
13. Toral $M$, Gómez-Guzmán $M$, Jiménez R, Romero $M$, Zarzuelo MJ, Utrilla MP, et al. Chronic peroxisome proliferator-activated receptor $\beta / \delta$ agonist GW0742 prevents hypertension, vascular inflammatory and oxidative status, and endothelial dysfunction in diet-induced obesity. Journal of hypertension. 2015;33(9):1831-44. Epub 2015/07/07. doi: 10.1097/hjh.0000000000000634. PubMed PMID: 26147382.

14. Chen D, Dixon BJ, Doycheva DM, Li B, Zhang Y, Hu Q, et al. IRE1a inhibition decreased TXNIP/NLRP3 inflammasome activation through miR-17-5p after neonatal hypoxic-ischemic brain injury in rats. Journal of neuroinflammation. 2018;15(1):32. Epub 2018/02/06. doi: 10.1186/s12974-018-1077-9. PubMed PMID: 29394934; PubMed Central PMCID: PMCPMC5797348.

15. Prentice H, Gharibani PM, Ma Z, Alexandrescu A, Genova R, Chen PC, et al. Neuroprotective Functions Through Inhibition of ER Stress by Taurine or Taurine Combination Treatments in a Rat Stroke Model. Advances in experimental medicine and biology. 2017;975 Pt 1:193-205. Epub 2017/08/30. doi: 10.1007/978-94-024-1079-2_17. PubMed PMID: 28849455.

16. Yin Y, Sun G, Li E, Kiselyov K, Sun D. ER stress and impaired autophagy flux in neuronal degeneration and brain injury. Ageing research reviews. 2017;34:3-14. Epub 2016/09/07. doi: 10.1016/j.arr.2016.08.008. PubMed PMID: 27594375; PubMed Central PMCID: PMCPMC5250579.

17. Zhu Y, Yu J, Gong J, Shen J, Ye D, Cheng D, et al. PTP1B inhibitor alleviates deleterious microglial activation and neuronal injury after ischemic stroke by modulating the ER stress-autophagy axis via PERK signaling in microglia. Aging. 2021;13(3):3405-27. Epub 2021/01/27. doi: 10.18632/aging.202272. PubMed PMID: 33495405; PubMed Central PMCID: PMCPMC7906217.

18. Harvey LD, Yin Y, Attarwala IY, Begum G, Deng J, Yan HQ, et al. Administration of DHA Reduces Endoplasmic Reticulum Stress-Associated Inflammation and Alters Microglial or Macrophage Activation in Traumatic Brain Injury. ASN neuro. 2015;7(6). Epub 2015/12/20. doi: 10.1177/1759091415618969. PubMed PMID: 26685193; PubMed Central PMCID: PMCPMC4710127.

19. Zhu Q, Liu Z, Wang Y, Song E, Song Y. Endoplasmic reticulum stress manipulates autophagic response that antagonizes polybrominated diphenyl ethers quinone induced cytotoxicity in microglial BV2 cells. Journal of hazardous materials. 2021;411:124958. Epub 2021/01/16. doi: 10.1016/j.jhazmat.2020.124958. PubMed PMID: 33450633.

20. Carloni S, Favrais G, Saliba E, Albertini MC, Chalon S, Longini M, et al. Melatonin modulates neonatal brain inflammation through endoplasmic reticulum stress, autophagy, and miR-34a/silent information regulator 1 pathway. Journal of pineal research. 2016;61(3):370-80. Epub 2016/07/22. doi: 10.1111/jpi.12354. PubMed PMID: 27441728.

21. Jarius S, Ruprecht K, Kleiter I, Borisow N, Asgari N, Pitarokoili K, et al. MOG-IgG in NMO and related disorders: a multicenter study of 50 patients. Part 2: Epidemiology, clinical presentation, radiological and laboratory features, treatment responses, and long-term outcome. Journal of neuroinflammation. 2016;13(1):280. Epub 2016/10/30. doi: 10.1186/s12974-016-0718-0. PubMed PMID: 27793206; PubMed Central PMCID: PMCPMC5086042. 
22. Liu SH, Yang CN, Pan HC, Sung YJ, Liao KK, Chen WB, et al. IL-13 downregulates PPAR-gamma/heme oxygenase-1 via ER stress-stimulated calpain activation: aggravation of activated microglia death. Cellular and molecular life sciences : CMLS. 2010;67(9):1465-76. Epub 2010/03/12. doi: 10.1007/s00018-009-0255-4. PubMed PMID: 20221786.

23. Pan HC, Yang CN, Hung YW, Lee WJ, Tien HR, Shen CC, et al. Reciprocal modulation of C/EBP-a and C/EBP- $\beta$ by IL-13 in activated microglia prevents neuronal death. European journal of immunology. 2013;43(11):2854-65. Epub 2013/07/25. doi: 10.1002/eji.201343301. PubMed PMID: 23881867.

24. Romeo-Guitart D, Leiva-Rodríguez T, Espinosa-Alcantud M, Sima N, Vaquero A, Domínguez-Martín H, et al. SIRT1 activation with neuroheal is neuroprotective but SIRT2 inhibition with AK7 is detrimental for disconnected motoneurons. Cell death \& disease. 2018;9(5):531. Epub 2018/05/12. doi: 10.1038/s41419-018-0553-6. PubMed PMID: 29748539; PubMed Central PMCID: PMCPMC5945655 under PCT extension.

25. Reiter RJ, Melchiorri D, Sewerynek E, Poeggeler B, Barlow-Walden L, Chuang J, et al. A review of the evidence supporting melatonin's role as an antioxidant. Journal of pineal research. 1995;18(1):1-11. Epub 1995/01/01. doi: 10.1111/j.1600-079x.1995.tb00133.x. PubMed PMID: 7776173.

26. Zhao D, Yu Y, Shen Y, Liu Q, Zhao Z, Sharma R, et al. Melatonin Synthesis and Function: Evolutionary History in Animals and Plants. Frontiers in endocrinology. 2019;10:249. Epub 2019/05/07. doi: 10.3389/fendo.2019.00249. PubMed PMID: 31057485; PubMed Central PMCID: PMCPMC6481276.

27. Ali T, Rahman SU, Hao Q, Li W, Liu Z, Ali Shah F, et al. Melatonin prevents neuroinflammation and relieves depression by attenuating autophagy impairment through FOXO3a regulation. Journal of pineal research. 2020;69(2):e12667. Epub 2020/05/07. doi: 10.1111/jpi.12667. PubMed PMID: 32375205.

28. Hardeland R. Melatonin and inflammation-Story of a double-edged blade. Journal of pineal research. 2018;65(4):e12525. Epub 2018/09/23. doi: 10.1111/jpi.12525. PubMed PMID: 30242884.

29. Shah SA, Khan M, Jo MH, Jo MG, Amin FU, Kim MO. Melatonin Stimulates the SIRT1/Nrf2 Signaling Pathway Counteracting Lipopolysaccharide (LPS)-Induced Oxidative Stress to Rescue Postnatal Rat Brain. CNS neuroscience \& therapeutics. 2017;23(1):33-44. Epub 2016/07/17. doi:

10.1111/cns.12588. PubMed PMID: 27421686; PubMed Central PMCID: PMCPMC6492734.

30. Taniguti EH, Ferreira YS, Stupp IJV, Fraga-Junior EB, Mendonça CB, Rossi FL, et al. Neuroprotective effect of melatonin against lipopolysaccharide-induced depressive-like behavior in mice. Physiology \& behavior. 2018;188:270-5. Epub 2018/02/20. doi: 10.1016/j.physbeh.2018.02.034. PubMed PMID: 29458118.

31. Carloni S, Riparini G, Buonocore G, Balduini W. Rapid modulation of the silent information regulator 1 by melatonin after hypoxia-ischemia in the neonatal rat brain. Journal of pineal research. 2017;63(3). Epub 2017/07/15. doi: 10.1111/jpi.12434. PubMed PMID: 28708259.

32. Choi SI, Kim KS, Oh JY, Jin JY, Lee GH, Kim EK. Melatonin induces autophagy via an mTORdependent pathway and enhances clearance of mutant-TGFBIp. Journal of pineal research. 2013;54(4):361-72. Epub 2013/02/01. doi: 10.1111/jpi.12039. PubMed PMID: 23363291. 
33. Choi SI, Lee E, Akuzum B, Jeong JB, Maeng YS, Kim TI, et al. Melatonin reduces endoplasmic reticulum stress and corneal dystrophy-associated TGFBIp through activation of endoplasmic reticulum-associated protein degradation. Journal of pineal research. 2017;63(3). Epub 2017/06/06. doi: 10.1111/jpi.12426. PubMed PMID: 28580641.

34. Chung SY, Han SH. Melatonin attenuates kainic acid-induced hippocampal neurodegeneration and oxidative stress through microglial inhibition. Journal of pineal research. 2003;34(2):95-102. Epub 2003/02/04. doi: 10.1034/j.1600-079x.2003.00010.x. PubMed PMID: 12562500.

35. Han L, Wang H, Li L, Li X, Ge J, Reiter RJ, et al. Melatonin protects against maternal obesityassociated oxidativestress and meiotic defects in oocytes via the SIRT3-SOD2-dependent pathway. Journal of pineal research. 2017;63(3). Epub 2017/06/29. doi: 10.1111/jpi.12431. PubMed PMID: 28658527.

36. Wu SM, Lin WY, Shen CC, Pan HC, Keh-Bin W, Chen YC, et al. Melatonin set out to ER stress signaling thwarts epithelial mesenchymal transition and peritoneal dissemination via calpain-mediated C/EBP $\beta$ and NFKB cleavage. Journal of pineal research. 2016;60(2):142-54. Epub 2015/10/31. doi: 10.1111/jpi.12295. PubMed PMID: 26514342.

37. Sheahan TD, Siuda ER, Bruchas MR, Shepherd AJ, Mohapatra DP, Gereau RWt, et al. Inflammation and nerve injury minimally affect mouse voluntary behaviors proposed as indicators of pain. Neurobiology of pain (Cambridge, Mass). 2017;2:1-12. Epub 2017/10/28. doi: 10.1016/j.ynpai.2017.09.001. PubMed PMID: 29075674; PubMed Central PMCID: PMCPMC5653321.

38. Hardeland R. Melatonin, noncoding RNAs, messenger RNA stability and epigenetics-evidence, hints, gaps and perspectives. International journal of molecular sciences. 2014;15(10):18221-52. Epub 2014/10/14. doi: 10.3390/ijms151018221. PubMed PMID: 25310649; PubMed Central PMCID: PMCPMC4227213.

39. Hardeland R. Melatonin and the pathologies of weakened or dysregulated circadian oscillators. Journal of pineal research. 2017;62(1). Epub 2016/10/21. doi: 10.1111/jpi.12377. PubMed PMID: 27763686.

40. Hardeland R. Recent Findings in Melatonin Research and Their Relevance to the CNS. Central nervous system agents in medicinal chemistry. 2018;18(2):102-14. Epub 2018/06/01. doi: 10.2174/1871524918666180531083944. PubMed PMID: 29848280.

41. Arioz BI, Tastan B, Tarakcioglu E, Tufekci KU, Olcum M, Ersoy N, et al. Melatonin Attenuates LPSInduced Acute Depressive-Like Behaviors and Microglial NLRP3 Inflammasome Activation Through the SIRT1/Nrf2 Pathway. Frontiers in immunology. 2019;10:1511. Epub 2019/07/23. doi: 10.3389/fimmu.2019.01511. PubMed PMID: 31327964; PubMed Central PMCID: PMCPMC6615259.

42. Ali T, Hao Q, Ullah N, Rahman SU, Shah FA, He K, et al. Melatonin Act as an Antidepressant via Attenuation of Neuroinflammation by Targeting Sirt1/Nrf2/HO-1 Signaling. Frontiers in molecular neuroscience. 2020;13:96. Epub 2020/07/01. doi: 10.3389/fnmol.2020.00096. PubMed PMID: 32595452; PubMed Central PMCID: PMCPMC7304371. 
43. González-Fernández B, Sánchez DI, Crespo I, San-Miguel B, de Urbina JO, González-Gallego J, et al. Melatonin Attenuates Dysregulation of the Circadian Clock Pathway in Mice With CCl(4)-Induced Fibrosis and Human Hepatic Stellate Cells. Frontiers in pharmacology. 2018;9:556. Epub 2018/06/13. doi: 10.3389/fphar.2018.00556. PubMed PMID: 29892224; PubMed Central PMCID: PMCPMC5985434.

44. Paterniti I, Campolo M, Cordaro M, Impellizzeri D, Siracusa R, Crupi R, et al. PPAR-a Modulates the Anti-Inflammatory Effect of Melatonin in the Secondary Events of Spinal Cord Injury. Molecular neurobiology. 2017;54(8):5973-87. Epub 2016/10/01. doi: 10.1007/s12035-016-0131-9. PubMed PMID: 27686077.

45. Kato H, Tanaka G, Masuda S, Ogasawara J, Sakurai T, Kizaki T, et al. Melatonin promotes adipogenesis and mitochondrial biogenesis in 3T3-L1 preadipocytes. Journal of pineal research. 2015;59(2):267-75. Epub 2015/07/01. doi: 10.1111/jpi.12259. PubMed PMID: 26123001.

46. Le PH, Kuo CJ, Cheng HT, Wu RC, Chen TH, Lin CJ, et al. Melatonin Attenuates Acute PancreatitisInduced Liver Damage Through Akt-Dependent PPAR-y Pathway. The Journal of surgical research. 2019;236:311-8. Epub 2019/01/30. doi: 10.1016/j.jss.2018.11.056. PubMed PMID: 30694771.

\section{Tables}

Due to technical limitations, tables are only available as a download in the Supplemental Files section.

\section{Figures}




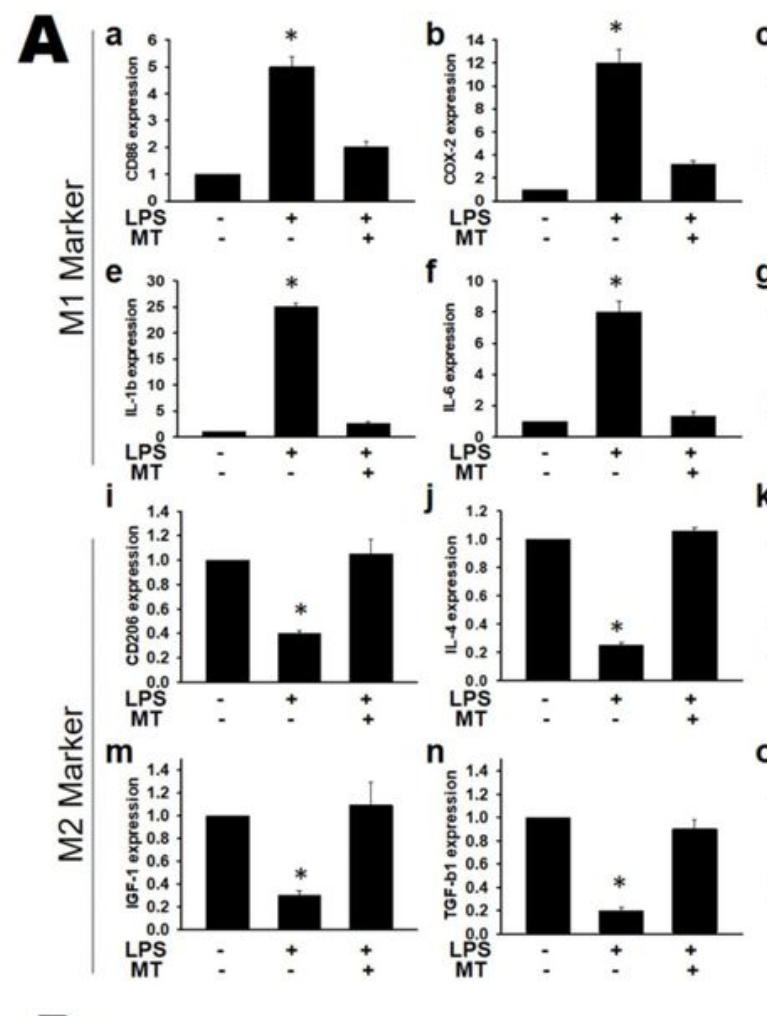

B a

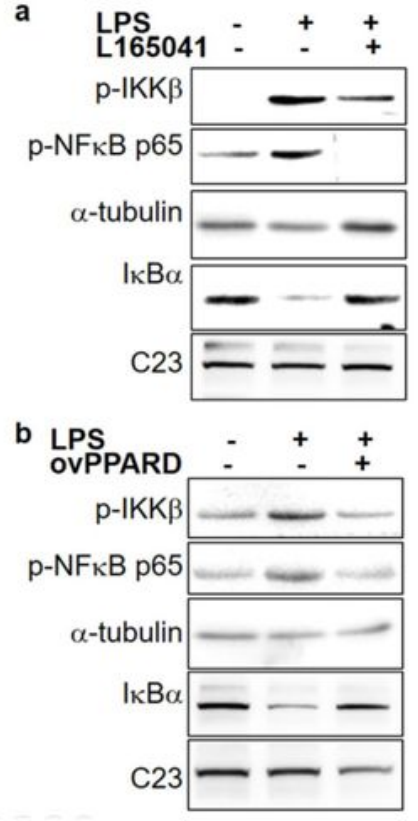

C

e
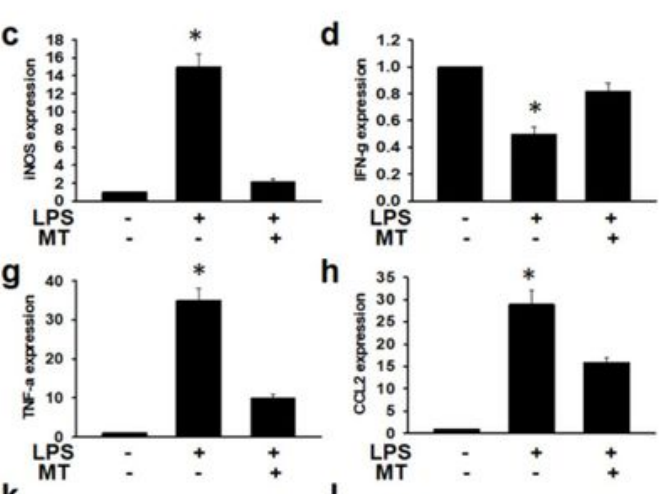

$k$
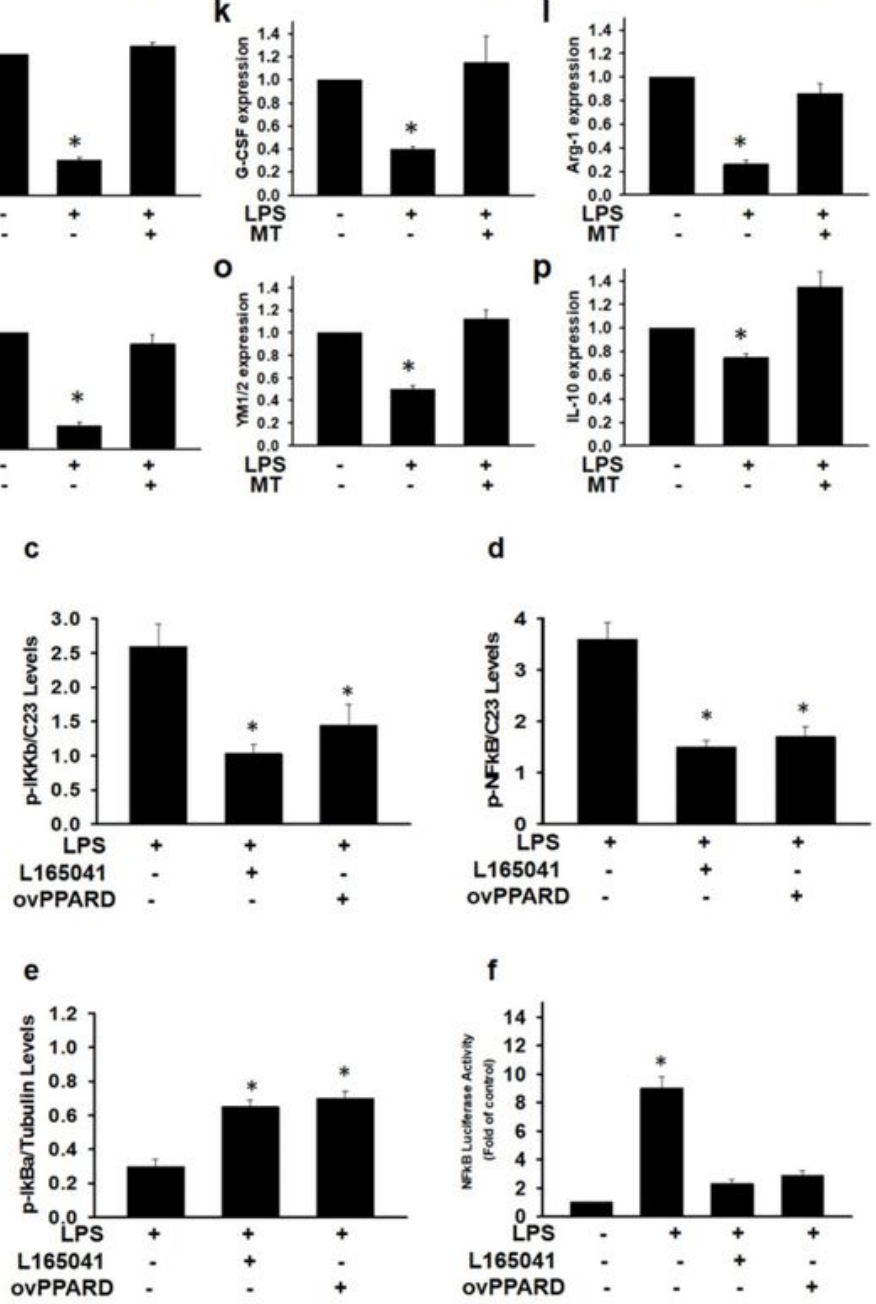

Fig.1

Figure 1

The prevention of LPS-evoked M1 microglial activation and predisposition to M2 polarization by melatonin. Microglia were cultured with Melatonin $(0.1 \mathrm{mM})$ followed by the incubation with LPS $(0.1$ $\mathrm{mg} / \mathrm{ml}$ ) for $24 \mathrm{hr}$. Melatonin suppressed the amoeboid "activated" morphology of microglia triggered by LPS. 
(1Aa-h) The Melatonin counteracted LPS-induced high mRNA expressions in M1 markers, such as CD86, COX-2, iNOS, IFNg, IL-1b, IL-6, TNF-a, and CCL2.

(1Ai-p) mRNA expressions of M2 markers (CD206, IL-4, GM-CSF, Arginase-1, IGF-1, TGF-b1, YM1/2, IL-10) in microglial cells.

(1Ba-b) Protein expressions in activated microglial cells as determined by Western blotting. Specific antibody probe as indicated for $\mathrm{p}-\mathrm{IKK}$, $\mathrm{p}-\mathrm{NFkB}$, IKB.

(1Bce) The quantification analysis for Western blotting images, and normalized with C23 or a-tubulin.

(1Bf) NFkB promoter luciferase activity. The functional activity was examined using the dual luciferase reporter assay kit. The result is a two-signal assay system that enables normalization of experimental signal (firefly luciferase) to a constitutively expressed control (Renilla luciferase). All results are presented as mean $\pm S D, n \geq 5,{ }^{*}<0.05$. compared to the Control group. 

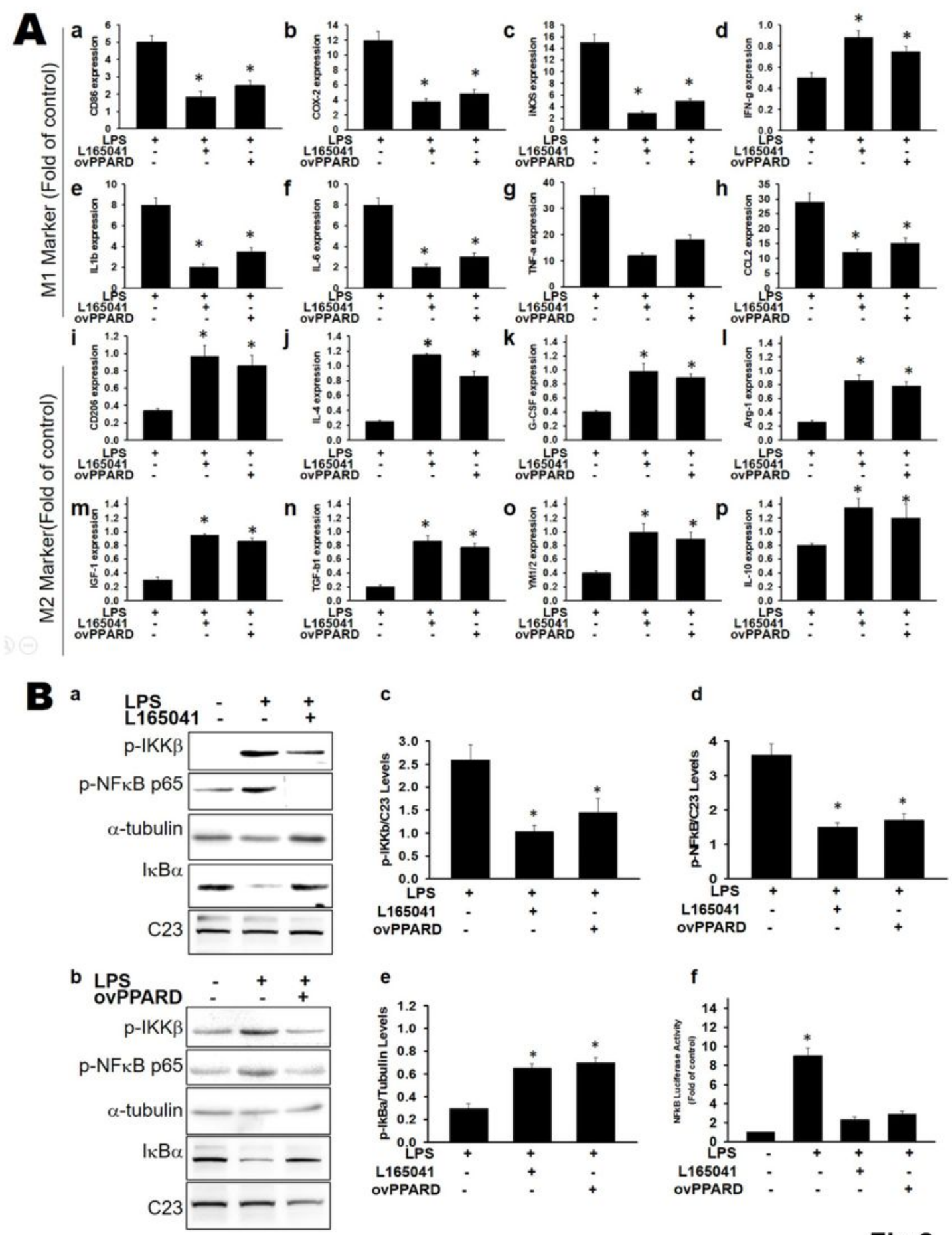

c
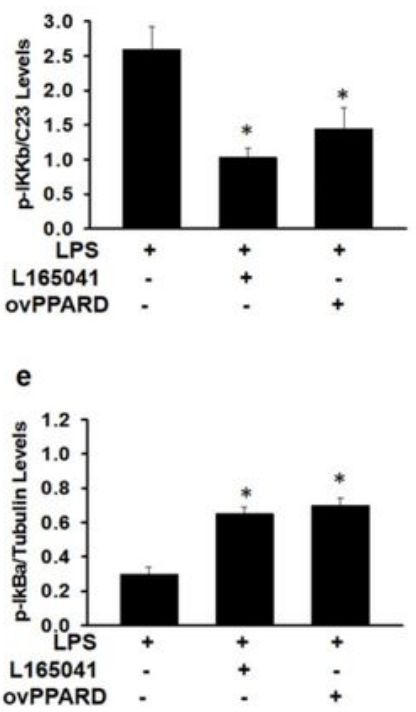

d
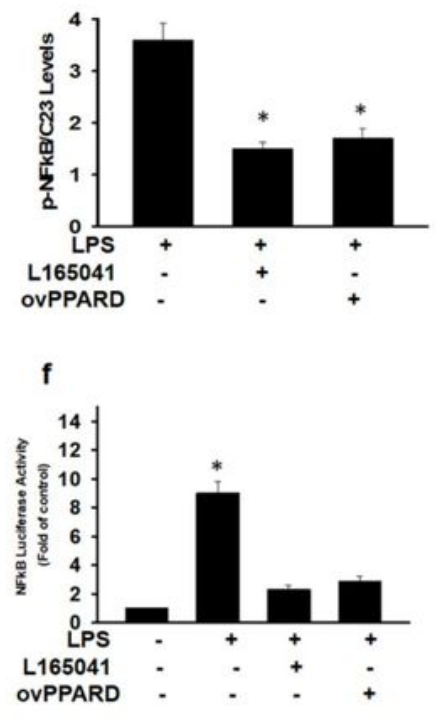

Fig.2

Figure 2

Agonizing PPARd either using L16504, or overexpression of PPARd switches the polarization from M1 to M2 in primary cultured microglia. Microglia were pretreated with PPARd agonist L16504, $(20 \mathrm{mM})$ or transfection PPARd plasmid (ovPPARd $5 \mathrm{mg} / \mathrm{ml}$ ) and then incubated with LPS $(0.1 \mathrm{mg} / \mathrm{ml})$ for $24 \mathrm{hr}$. 
(2Ba-h) The L16504 or ovPPARd reversed LPS-induced mRNA expressions of M1 markers, such as CD86, Cox-2, iNOS, IFNg, IL-1a, IL-6, TNF-a, and CCL2.

(2Ai-p) mRNA expressions of M2 markers (CD206, IL-4, GM-CSF, Arginase-1, IGF-1, TGF-b1, YM1/2, IL-10) in microglial cells.

(2Ba-b) Protein expressions in activated microglial cells as determined by Western blotting. Specific antibody probe as indicted for $\mathrm{p}-\mathrm{IKK}$, p-NFkB, IKB.

(2Bc-e) The quantification analysis for Western blotting images, and normalized with C23 or a-tubulin.

(2Bf) NFkB promoter luciferase activity. The functional activity was examined using the dual luciferase reporter assay kit and presented as ratio of normalization of experimental signal (firefly luciferase) to a constitutively expressed control (Renilla luciferase). All data are presented as mean $\pm S D, n \geq 5,{ }^{*}<0.05$. compared to the Control group.
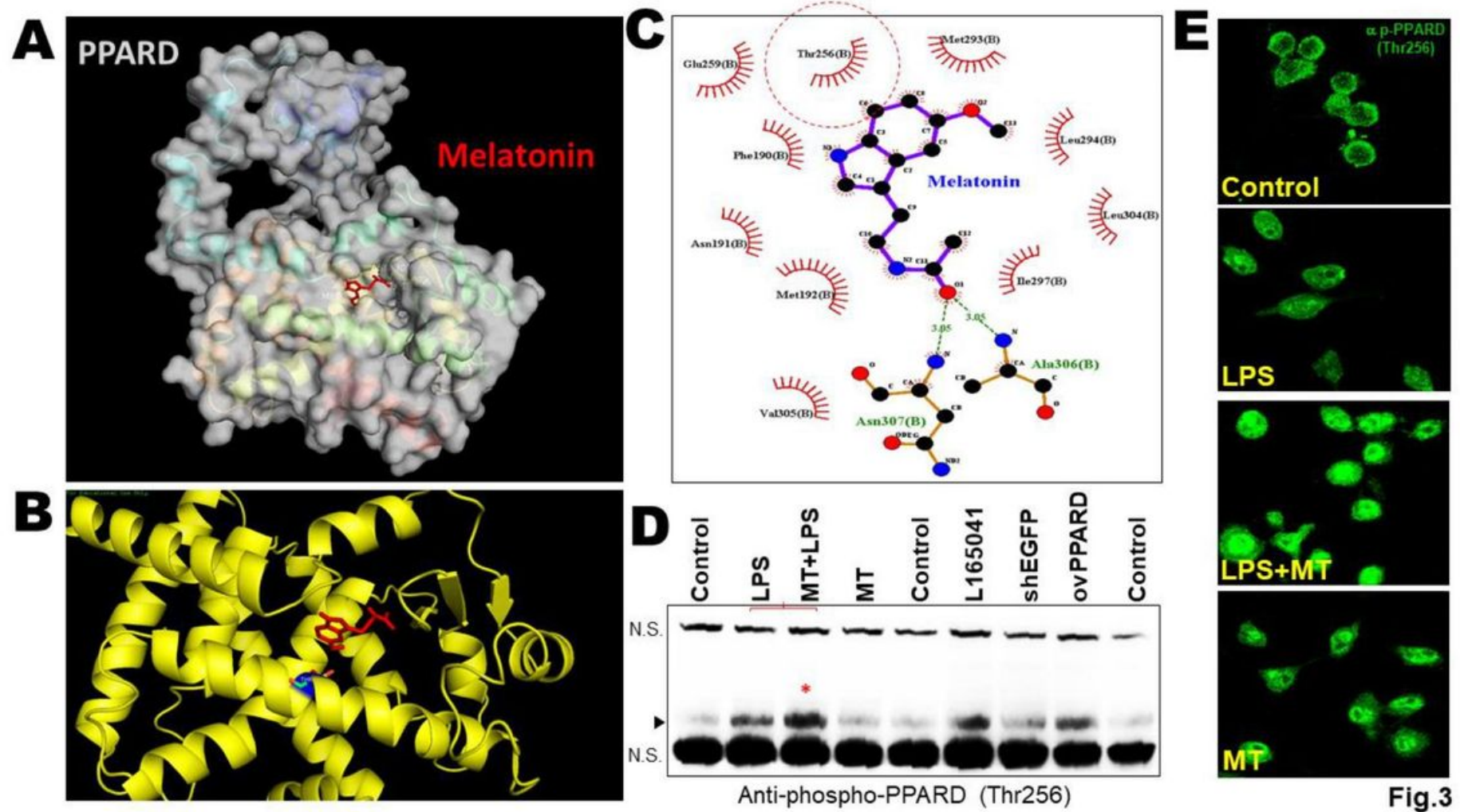

Fig.3

Figure 3

Molecular docking, dynamic simulation studies and biochemical evidence identified activation properties by Melatonin targeting PPARd. General and local overviews of the best interaction after automated docking of binding Melatonin (red) to the active site of PPARd. 
(A) Surface representation of the ligand-receptor binding. Melatonin and PPARd are shown in surface representation. The close depiction of the active region of target protein with embedded Melatonin. Melatonin (red) targets PPARd 3D structure in gray color with interior color having surface format. The cave entrance of the zoom-in binding mode of PPARd and Melatonin are shown.

(B) Docking interaction between Melatonin and target protein (PPARd). Local interaction positions of the HDAC active site. The molecular structure of human PPARd with Thr 256 is depicted as a ribbon diagram, with a-helices, b-pleated sheets, and loops. The binding mode of PPARd and Melatonin are shown targeting Thr 256 representation. The 3D representation of the interactions of Melatonin binding to the PPARd active site, as generated by PyMol.

(C) LIGPLOT generates schematic 2-D representations of protein-ligand complexes shown that interplay with candidate sites containing Thr256 residue. The hydrogen bonds were observed between Melatonin and PPARd amino acids.

(D) Microglial cells were pretreated with $0.1 \mathrm{mM}$ Melatonin, followed by treatment with LPS $0.1 \mathrm{mg} / \mathrm{ml}$ for 30 minutes. The phospho-PPARd (Thr256) protein levels were analyzed by Western blotting.

(E) Microglial cells grown on coverslips were induced by LPS $(0.1 \mathrm{mg} / \mathrm{ml})$ with or without Melatonin $(0.1$ $\mathrm{mM}$ ) for $1 \mathrm{~h}$, and then incubated and fixed with monoclonal antibodies targeted on PPARd. The method for immunofluorescence staining for PPARd was depicted in the text. The majority of the nuclei in primary microglia treated by Melatonin or combined LPS/Melatonin were PPARd staining positive. Data are executed at least four independent experiments. 

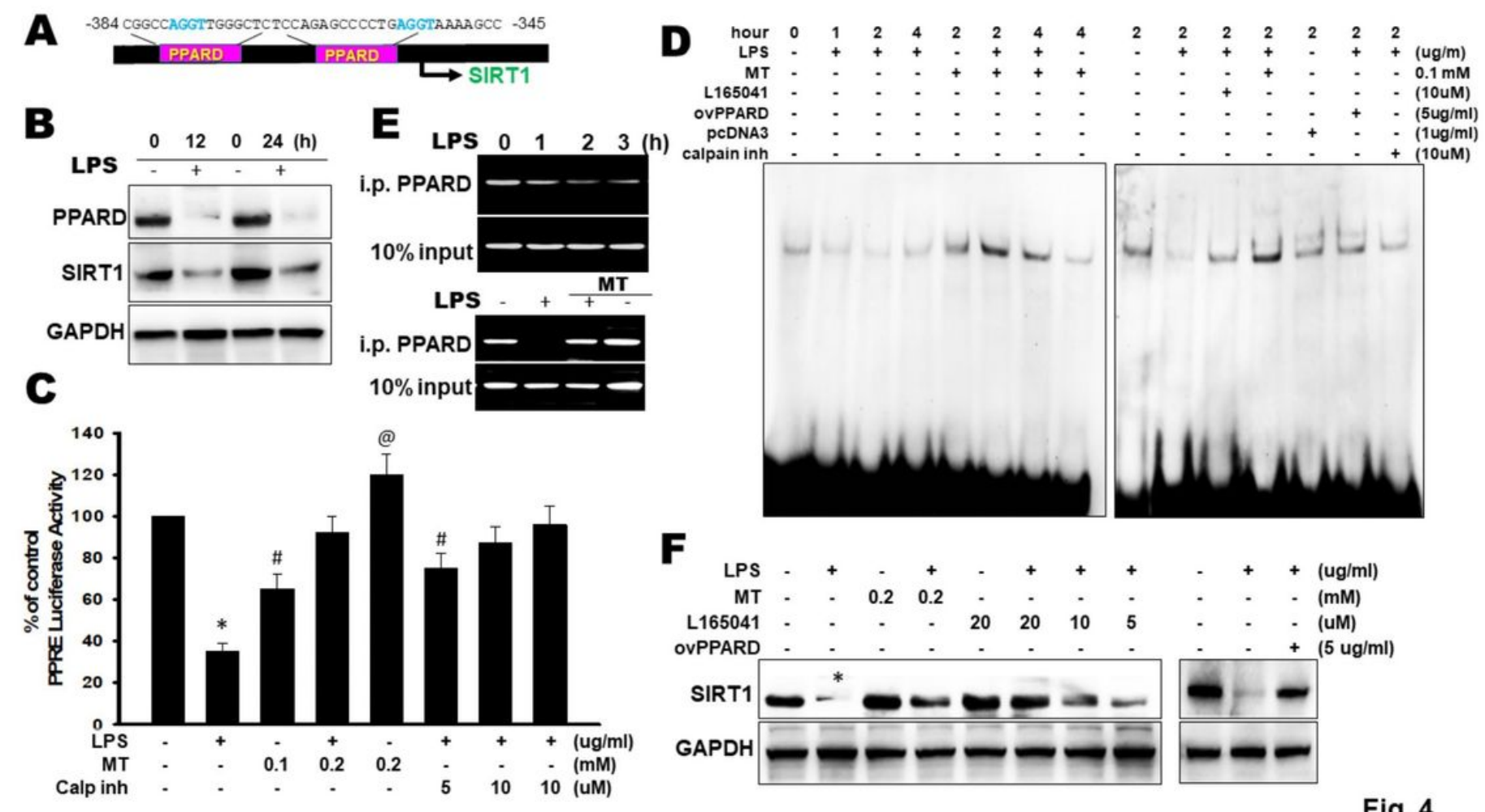

Figure 4

Melatonin-evoked PPARd activity regulated SIRT1 production.

(A) The sequences of PPARd on theSIRT1 promoter region were shown, pointing out the sub fragments which have been used in this study (underlined). Promoter regulation in the SIRT1 promoter-flanking region contains the cis-acting elements PPARd (-345 to-384) DNA binding site.

(B) The culture of primary microglia was conditioned in serum-free medium overnight either presence or absence of LPS-treated $(100 \mathrm{ng} / \mathrm{ml})$ status. Whole cell lysates were blotted and immunostained with antibodies against PPARd and SIRT1.

(C) The cells were transiently transfected with the PPRE luciferase reporter plasmid followed by thymidine kinase promoter-driven Renilla-luciferase vector, which were pre-treated with Melatonin or calpain inhibitors (Z-Leu-Leu-CHO; $10 \mathrm{mM}$ ). The relative activity was measured in luciferase assay as described in the text. The luciferase activities in means \pm SD were calculated by triplicate determinations.

(D) The synthetic dsDNA representing PPARd-binding sites in the promoter of SIRT1 gene was analyzed by electrophoretic mobility shift assay (EMSA) using nuclear extracts in control or LPS groups following stimulation of cells with or without Melatonin. The unbound excess radiolabeled probe migrated out of the gel and shown in the bottom. Cells were treated with Melatonin at various time frame, and nuclear PPARd DNA binding activity was analyzed by EMSA. Cells were pretreated with L165041, transfected with 
ovPPARd, pcDNA3 or calpain inhibitor followed by stimulation with LPS for 1-4 $\mathrm{h}$. At least three independent measurements were done for each data. Arrow points specific PPARd sequences located on SIRT1 promoter DNA binding site. The PPARd DNA binding labeled probe as indicated. All experiments were repeated at least three times.

(E) PPARd binds to the SIRT1 promoter in vivo. ChIP assay was carried out in control, LPS or Melatonin. After formaldehyde cross-linking, the PPARd-binding DNA fragments were recovered by immunoprecipitation using PPARd antibodies. Purified precipitates and input DNA were analyzed by PCR using primers specific for SIRT1 promoters. The cross-links were reversed, and the recovered DNA population was analyzed by PCR with primers designed for detecting SIRT1. An amplified PCR fragment is visible in the DNA immunoprecipitated with PPARd antibody in SIRT1 promoters. There is a subtle detection of PPARd band in the LPS-treated groups which are reversed by Melatonin. PCR products were subjected to gel electrophoresis and visualized by ethidium bromide staining; $10 \%$ aliquot of the precleared chromatin was regarded as an input. This experiment has been replicated at least four times with independent assays. All experiments were repeated at least four times.

(F) Cells were exposed to Melatonin, L165041 or transfected with pcDNA3-PPARd, pcDNA3 for overexpression PPARd, followed by stimulation with LPS for $24 \mathrm{~h}$. Cells were evaluated by Western blotting. All experiments were repeated at least five times.

$\mathbf{A}$
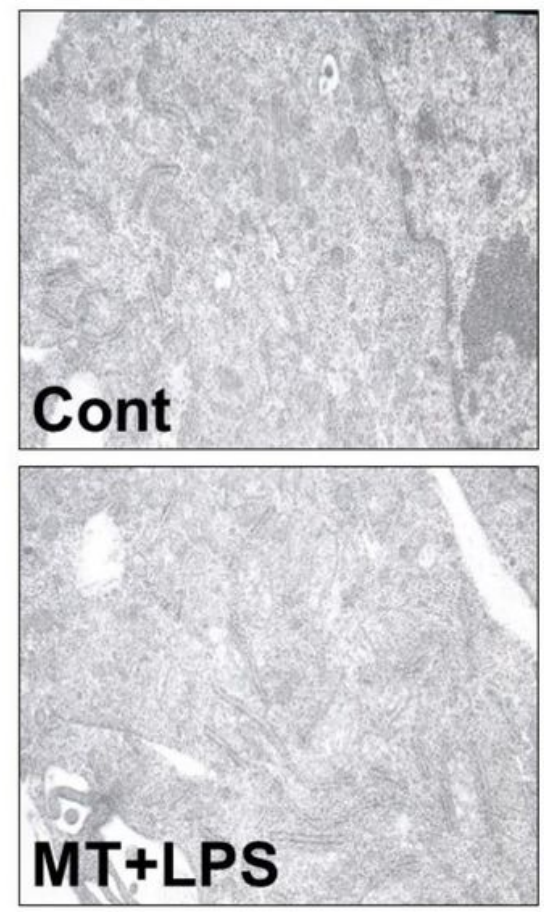
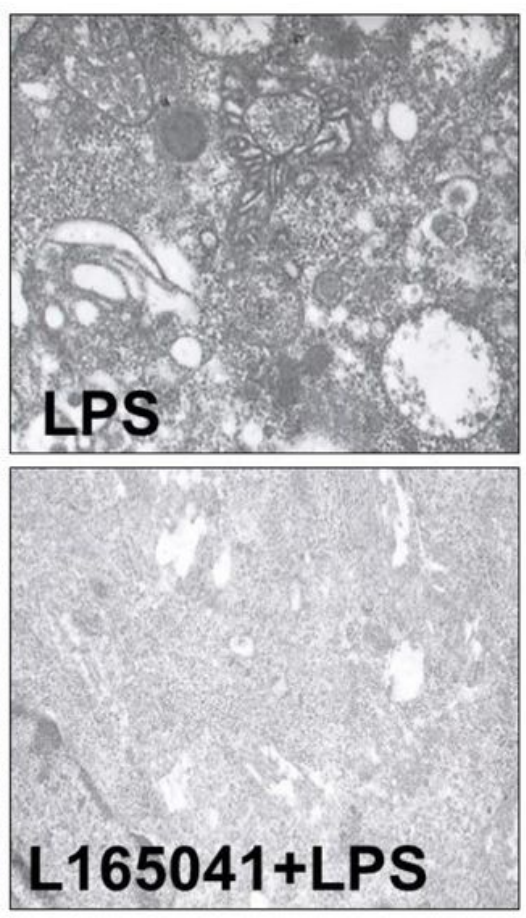

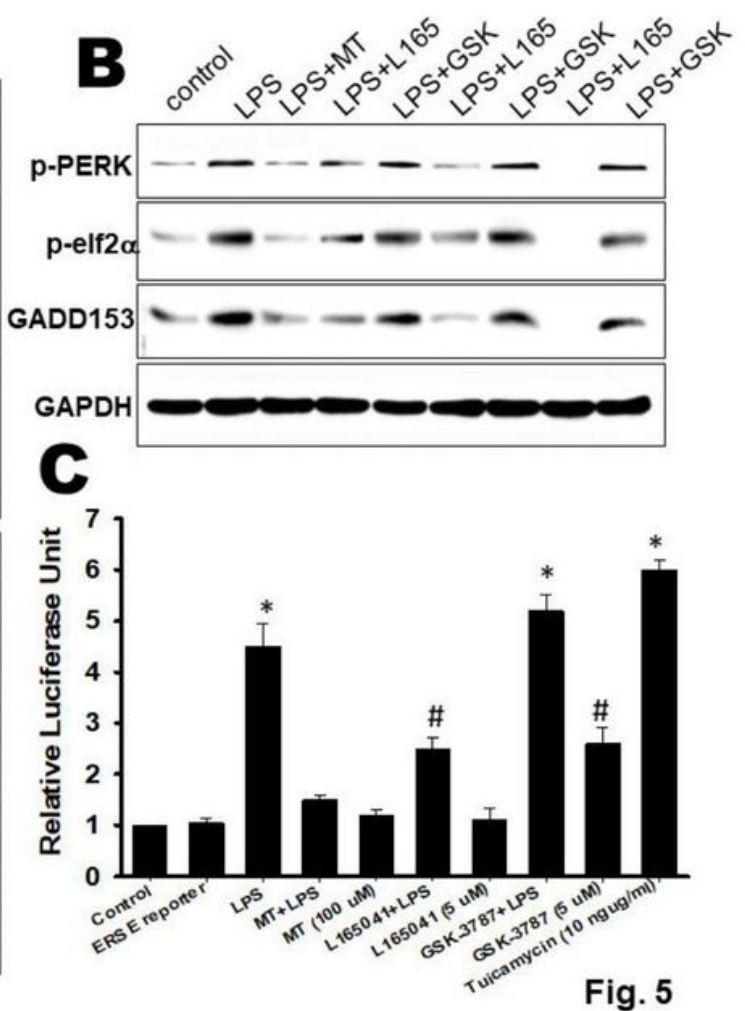

Fig. 5 
Figure 5

Melatonin thwarted the production of ER stress-related markers in LPS-activated microglia.

(A) Microglial cells were induced by LPS $(0.1 \mathrm{mg} / \mathrm{ml})$ in the presence or absence of Melatonin, L165041 for18 h. Cells were obtained and visualized by electron microscopy which were depicted in methodology section. The representative of three independent experiments was conducted in each measurement; $b$ : LPS-treated cell displays ER dilation photography; c: Cells treated by Melatonin+LPS; d: Combination of L165041 and LPS reduces serious ER dilation with the typical imaging of decreased distention and a fragmented organelle; Arrows indicate dilated ER. Original magnifications: $9800 \mathrm{~K}$

(B) Primary microglia were cultured in serum-free medium for $16 \mathrm{~h}$ at the presence or absence of LPS (100 ng/ml) with or without Melatonin, PPARd agonist L165041, or PPARd antagonist GSK3787 (5-20 $\mathrm{mM}$ ). Cell lysates were immunostained and blotted with antibodies for phospho-PPERK, phospho-elf2-a, and GADD153. (C) Primary rat microglial cells were co-treated with LPS (100 ng/ml) and Melatonin, PPARd agonist L165041, or PPARd antagonist GSK3787 or $18 \mathrm{~h}$, and cell lysates were used to measure ERSE luciferase activity. Tunicamycin treatment taken as a positive control regarded as an ER stress igniter. Each experiment was repeated at least five times.

A

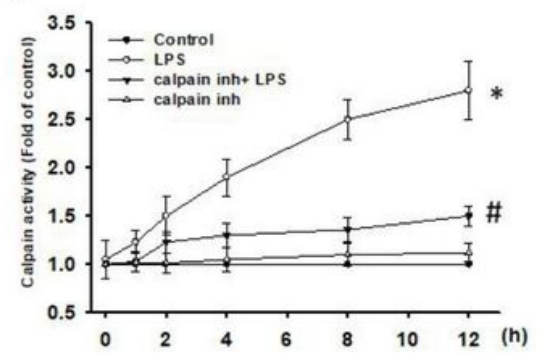

B
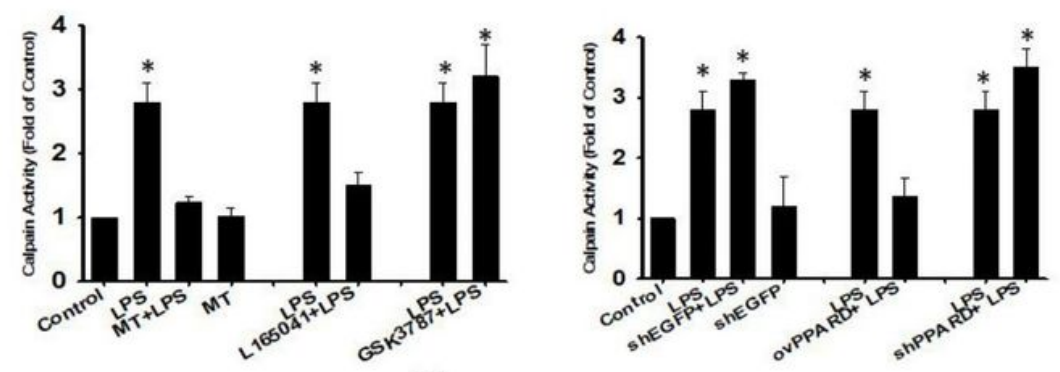

F

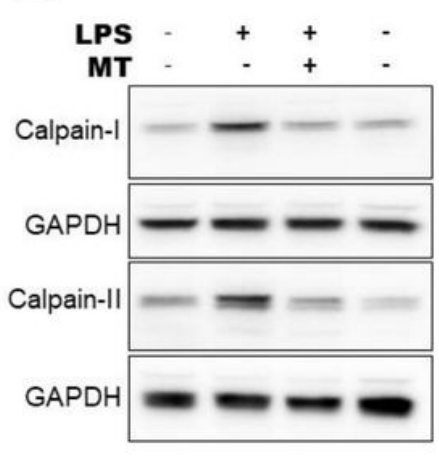

E

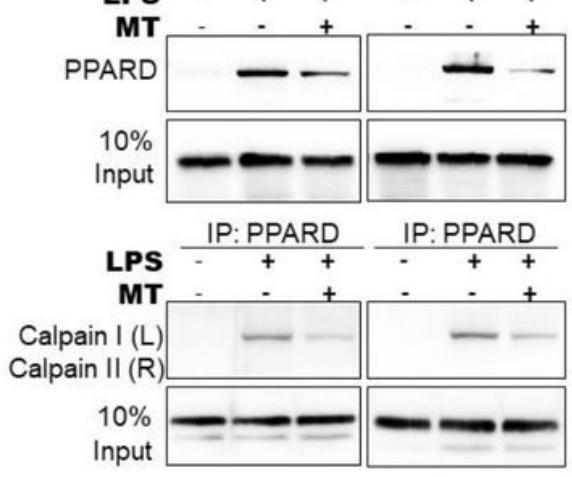

C

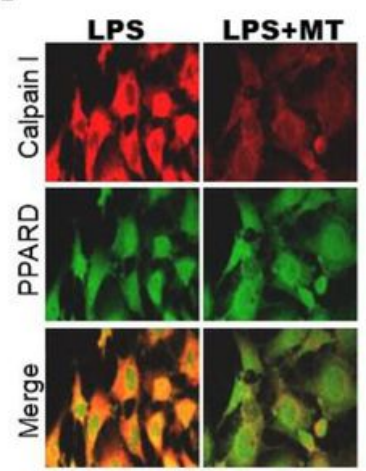

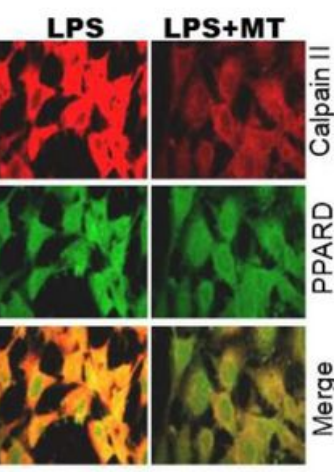

Fig.6

Figure 6 


\section{Melatonin reduced calpain activation in LPS-activated microglia.}

Primary microglia were cultured in serum-free medium for $18 \mathrm{~h}$ at the presence or absence of LPS $(0.1$ $\mathrm{mg} / \mathrm{ml}$ ) with or without calpain inhibitor Z-Leu-Leu-CHO (10 mM), Melatonin (100 mM) and L165041 (20 $\mathrm{mM}$ ) for 0.5-12 h. Calpain activity was calculated with the fluorescent calpain substrate Suc-LLVY-AMC.

(A) Presented induction time course depend effect.

(B) Cell pretreatment as indicated, further to examine in 12 hours.

(C) Transfection with shEGFP, shPPARd, ovPPARd overnight, and following LPS induction.

(D) Whole cell lysates were blotted and immunostained with antibodies against calpain-I and calpain-II at 24 hours. The data are presented as means $\pm S D(n=5)$. ${ }^{P}<0.05$ as compared with control; \#P $<0.05$ as compared with LPS alone.

(E) Interaction of calpain and PPARd were detected in primary microglia. Immunoprecipitated proteins were obtained, subjected to SDS-PAGE and immunoblotted with anti-calpain-I, anti-calpain-II or antiPPARd antibodies. The results were conducted at least three independent experiments. Measurements are exhibited as means $\pm S D(n=5)$. ${ }^{*}<0.05$ as compared with control; \#P $<0.05$ as compared with LPS alone.

(F) Primary antibodies for calpain-II and PPARd were administrated to the targets cells followed by secondary antibodies coupled with FITC conjugated (green) or tetramethyl rhodamine isothiocyanate (TRITC)-conjugated (red), respectively. Co-localization of two labeled antigens was detected as a single imaging when the imaging from both channels was overlaid. (red: calpain-I,-II; green: PPARd) The data are representative of at least three independent experiments. The data are presented as means $\pm S D(n=5)$. *P $<0.05$ as compared with control; \#P $<0.05$ as compared with LPS alone. 

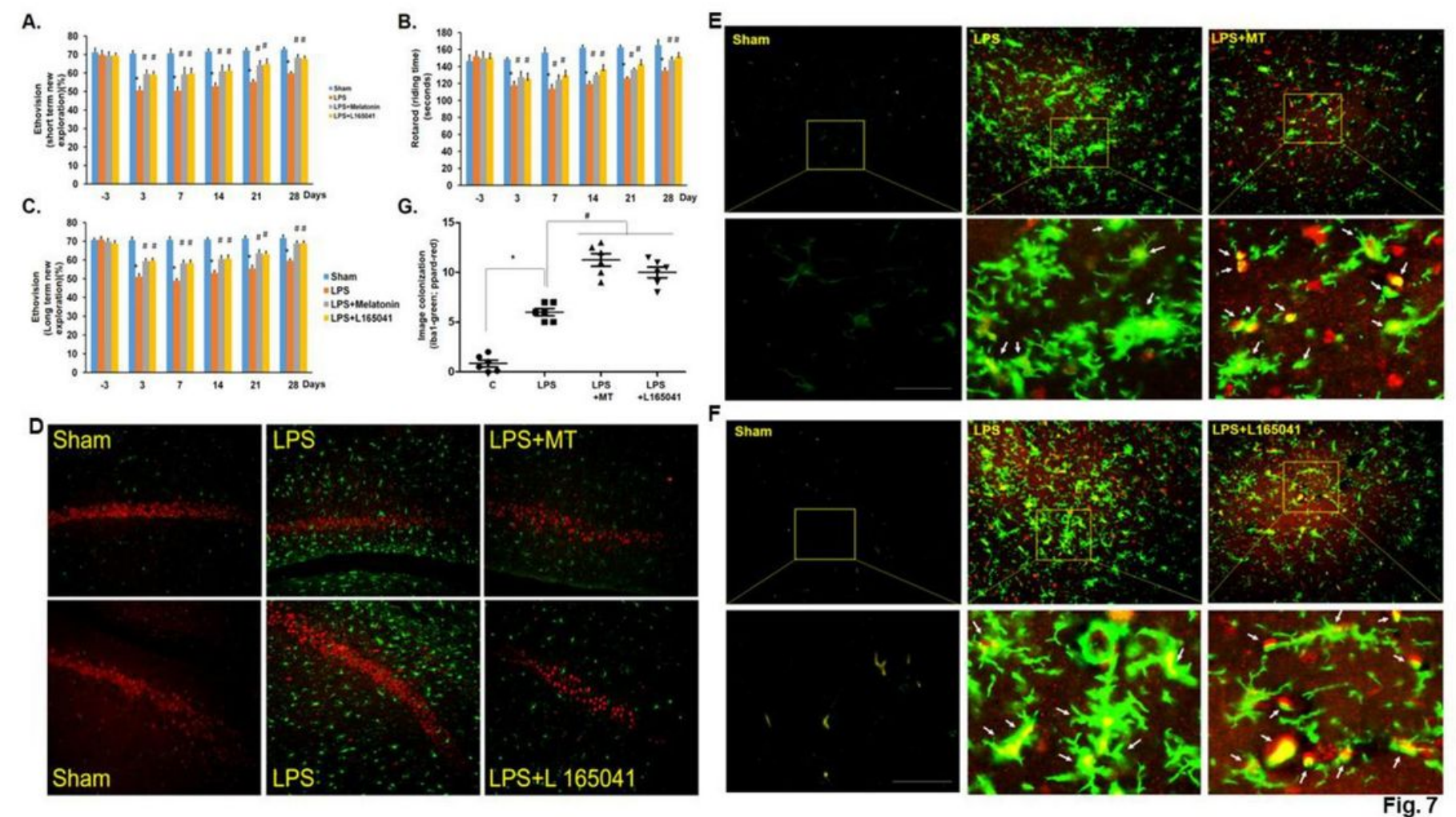

\section{Figure 7}

Neurobehavior assessment after intracerebral injection of melatonin and L 165041. In experimental design of neuro-inflammation model, animals received the neurobehavior assessment 3 days before intracerebral injection and then $3,7,14,21$, and 28 days after operation. Mice were intravenously injected with or without Melatonin ( $5 \mathrm{mg} / \mathrm{kg}$ ), or L165041 (2 mg/kg) by i.p. injection or local pretreated at $24 \mathrm{hrs}$ after LPS-injection. Animal were sacrificed further to perform histological and immunofluorescence analysis at day 7 after induction. Melatonin $(5 \mathrm{mg} / \mathrm{kg})$, and L165041 $(2 \mathrm{mg} / \mathrm{kg})$ reduced activated microglia-induced aggravated volume as assessed by immunofluorescence staining.

(A) Upper panel, plot of the short term (1 hours) Ethovision new exploration (\%) in different treatment groups related to the different time points.

(B) Plots of riding time in Rotarod assessment (seconds) in the different treatment groups related to the different time frame. $\mathrm{N}=6$ Sham, LPS, LPS+melatonin, LPS+L165041: see text. Data: mean \pm SD ** indicated the $p$-value $<0.001$ with LPS group related to sham group. \#\# indicated the $p$-value $<0.001$ either with LPS+melatonin or LPS+L165041 related to LPS

(C) Lower panel, Plot of the long term (24 hours) Ethovision new exploration (\%) in different treatment groups related to the different time points 
(D) Pharmacological inhibition by Melatonin or L165041 markedly blocked activated microglia cells in brain injury.

(D) Low magnification 100X or (E-F) High magnification 1200X presented neural cells ( $\mathrm{NeuN}$, red) and activated microglia (Iba1, green).

(E-F) Primary antibodies for microglia marker Iba1 and PPARd were conjugated to microglia followed by secondary antibodies coupled with FITC conjugated (green) or TRITC-conjugated (red), respectively as indicated. Co-localization of two labeled antigens was detected as a single image when the images from both channels were overlaid. (red: PPARd; green: Iba1).

(G) Quantification analysis for confocal microscope image localization between microglia markers Iba1green; PPARd-red. The image in each section was measured in five images from each treatment under HFP $(n>5)$. All data are shown as means \pm SD of five independent experiments. ${ }^{*} P<.05$ compared to controls; \#P $<.05$ compared to LPS.

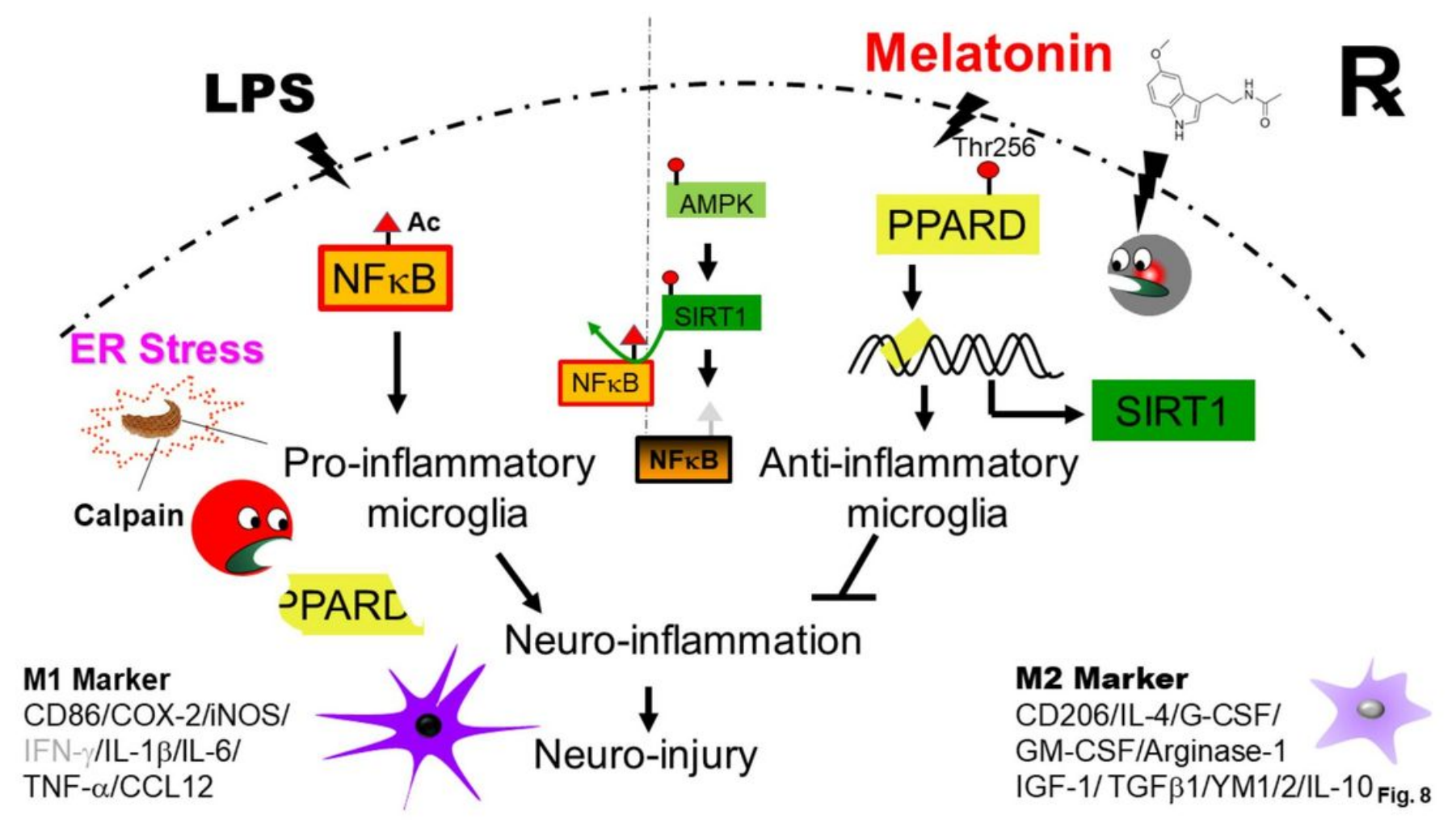

Figure 8

Schematic of proposed mechanism for the role of Melatonin in activated microglia and neuroinflammation effects. LPS-induced ER stress downregulated PPARd and NFkB activation, subsequently 
reduces SIRT1 expression and then triggers the microglia activation and brain damage. In the present study, we provide the evidence to demonstrate that melatonin plays a potential protective role in neuroprotective effects through PPARd/SIRT1 pathway. In addition, PPARd pharmacological agonists L165041 also possessed similar effects. These results suggest that the activation of PPARd/SIRT1 by melatonin could counteract the detrimental effect of LPS. Also, the results suggest melatonin may exert a therapeutic effect for neuroinflammatory disorders.

\section{Supplementary Files}

This is a list of supplementary files associated with this preprint. Click to download.

- Tables.docx 